\title{
Precisely Regulated Luminescent Gold Nanoparticles for Identification of Cancer Metastases
}

Yue Tan,${ }^{\dagger}$ Kui He,${ }^{\dagger}$ Bing Tang, ${ }^{\dagger}$ Huarui Chen,${ }^{\dagger}$ Zhipeng Zhao, ${ }^{\dagger}$ Chengqian Zhang, ${ }^{\dagger}$ Li Lin, ${ }^{\star}$ and Jinbin Liu ${ }^{*} \dagger$

$\dagger$ Key Laboratory of Functional Molecular Engineering of Guangdong Province,

School of Chemistry and Chemical Engineering, South China University of

Technology, Guangzhou 510640, China.*e-mail: cejbliu@scut.edu.cn

*Department of Oncology, Nanfang Hospital, Southern Medical University, Guangzhou, 510515, China.

The supporting information includes:

Table S1-3

Figure S1-28

Movie S1-2

Supporting References 
Supporting tables and figures

Table S1. Synthesis of ultrasmall luminescent AuNPs.

\begin{tabular}{cccc}
\hline $\begin{array}{c}\text { AuNPS } \\
\text { (HAuCl } \text { }_{4} / \text { PEG-SH/MSA-SH) }\end{array}$ & $\begin{array}{c}\text { EDC/NSH/MIZ-H } \\
\text { Reaction Time } \\
(\mathrm{h}) \text { at } 95{ }^{\circ} \mathrm{C}\end{array}$ \\
\hline MSA & $1: N A: 3$ & - & 24 \\
MIZ & $1: N A: 3$ & + & 24 \\
001PMIZ & $1: 0.01: 3$ & + & 22 \\
005PMIZ & $1: 0.05: 3$ & + & 22 \\
01PMIZ & $1: 0.1: 3$ & + & 20 \\
02PMIZ & $1: 0.2: 3$ & + & 18 \\
PEG & $1: 0.9:$ NA & - & 0.5 \\
\hline
\end{tabular}

The charge-reversal AuNPs: MIZ-AuNPs (MIZ), 001PMIZ-AuNPs (001PMIZ), 005PMIZ-AuNPs (005PMIZ), 01PMIZ-AuNPs (01PMIZ) and 02PMIZ-AuNPs (02PMIZ). The non-charge-reversal AuNPs: MSA-AuNPs (MSA) and PEG-AuNPs (PEG). NA, not available.

Table S2. The values of fluorescence quantum yield of the AuNPs.

\begin{tabular}{cc}
\hline & Quantum Yield (\%) \\
\hline MSA & 1.77 \\
MIZ & 1.79 \\
001PMIZ & 1.45 \\
005PMIZ & 1.38 \\
01PMIZ & 1.24 \\
02PMIZ & 1.19 \\
PEG & 0.72 \\
\hline
\end{tabular}

The fluorescence quantum yield of the AuNPs were consistent with those of the typically reported NIRemitting AuNPs. ${ }^{1}$ The MSA-AuNPs showed the highest fluorescence quantum yield, but the increase of PEG-SH might slightly decrease the quantum yields. However, the luminescence of all the AuNPs were highly observable through a commercially available in vivo imaging system.

Table S3. The values of fluorescence lifetime of the AuNPs.

\begin{tabular}{cccccc}
\hline & $\tau(\mu \mathrm{s})$ & $\tau_{1}(\mu \mathrm{s})$ & $\tau_{2}(\mu \mathrm{s})$ & $\mathrm{f}_{1}$ & $\chi^{2}$ \\
\hline MSA & 2.59 & 2.79 & 0.51 & 0.91 & 1.33 \\
MIZ & 2.64 & 2.87 & 0.55 & 0.90 & 1.12 \\
001PMIZ & 2.98 & 3.46 & 0.63 & 0.83 & 1.14 \\
005PMIZ & 3.43 & 4.00 & 0.72 & 0.83 & 1.04 \\
01PMIZ & 3.76 & 4.26 & 0.81 & 0.86 & 1.10 \\
02PMIZ & 4.12 & 4.79 & 0.90 & 0.83 & 1.24 \\
PEG & 1.92 & 2.08 & 0.25 & 0.91 & 1.38 \\
\hline
\end{tabular}

The ultrasmall AuNPs showed microsecond fluorescence lifetimes $(\tau)$ : MSA-AuNPs $(2.59 \mu \mathrm{s})$, MIZ-

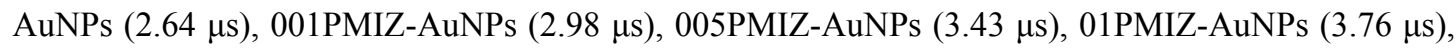
02PMIZ-AuNPs $(4.12 \mu \mathrm{s})$ and PEG-AuNPs $(1.92 \mu \mathrm{s})$, suggesting that the emissions were originated from the triplet excited states. 
Table S4. The migration distance differences $\left(\Delta d=d_{\mathrm{pH} 7.4}-d_{\mathrm{pH} 6.5}\right)$ of the charge-reversal AuNPs between pH 7.4 and 6.5 .

\begin{tabular}{cccc}
\hline AuNPs & $d_{\mathrm{pH} \mathrm{7.4}}(\mathrm{mm}) d_{\mathrm{pH} 6.5}(\mathrm{~mm})$ & $\Delta d(\mathrm{~mm})$ \\
\hline MIZ & $6.9 \pm 0.07$ & $2.8 \pm 0.04$ & $9.7 \pm 0.1$ \\
001PMIZ & $6.6 \pm 0.03$ & $3.2 \pm 0.07$ & $9.9 \pm 0.1$ \\
005PMIZ & $5.8 \pm 0.1$ & $4.1 \pm 0.07$ & $9.8 \pm 0.2$ \\
01PMIZ & $5.2 \pm 0.1$ & $4.6 \pm 0.03$ & $9.8 \pm 0.1$ \\
02PMIZ & $4.3 \pm 0.08$ & $5.3 \pm 0.06$ & $9.6 \pm 0.09$ \\
\hline
\end{tabular}

The charge reversal ability was evaluated by the migration distance differences $\left(\Delta d=d_{\mathrm{pH} 7.4}-d_{\mathrm{pH} 6.5}\right)$ between $\mathrm{pH} 6.5$ and $\mathrm{pH}$ 7.4. There was no significant difference in the charge-reversal ability among the charge-reversal AuNPs. The average value of $\Delta d$ was $9.8 \mathrm{~mm}$.

Table S5. The charge-reversal ability of charge-reversal AuNPs.

\begin{tabular}{cc}
\hline AuNPS & $\zeta_{\mathrm{pH} 6.5}-\zeta_{\mathrm{pH} 7.4}(\mathrm{mV})$ \\
\hline MIZ & $32.7 \pm 4.5$ \\
001PMIZ & $26.6 \pm 2.8$ \\
005PMIZ & $26.0 \pm 3.7$ \\
$01 \mathrm{PMIZ}$ & $28.6 \pm 1.3$ \\
02PMIZ & $30.0 \pm 2.6$ \\
\hline
\end{tabular}

The ability of charge reversal also was evaluated by the differences of $\zeta$-potential values $\left(\Delta \zeta=\zeta_{\mathrm{pH}} 6.5-\right.$ $\zeta_{\mathrm{pH}}$ 7.4) between $\mathrm{pH} 6.5$ and $\mathrm{pH}$ 7.4. There was no significant difference in the charge-reversal ability among these AuNPs. The average value of $\Delta \zeta$-potential was $28.8 \mathrm{mV}$. 
a

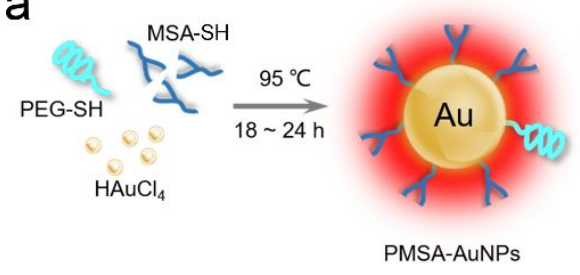

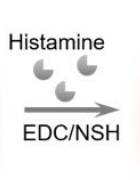

b

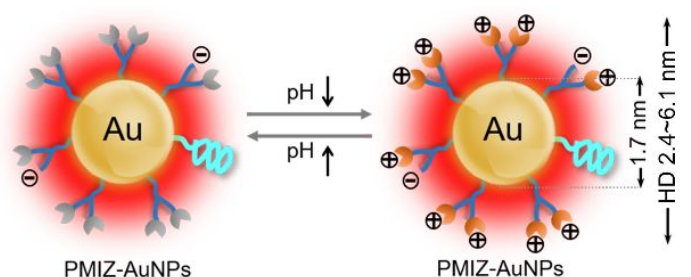

PMIZ-AuNPs

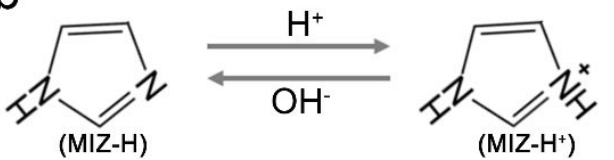

Figure S1. Synthesis of ultrasmall charge-reversal luminescent AuNPs with pH-responsive MIZH groups. a, The typical synthesis process. b, The structure of MIZ-H groups with $\mathrm{pH}$ changes.

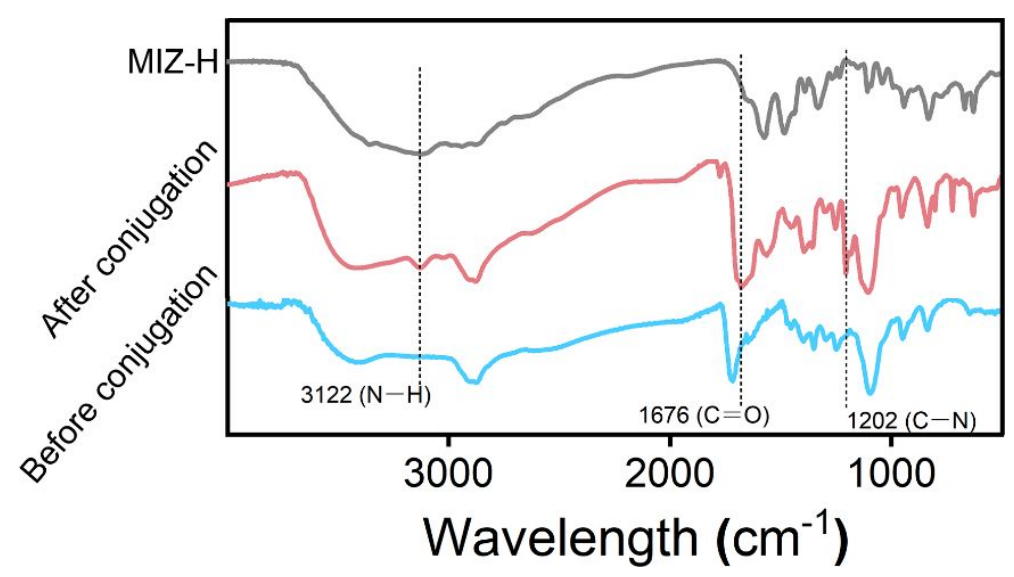

Figure S2. The FT-IR spectra of MIZ-H and 02PMIZ-AuNPs before and after conjugation of MIZH, respectively. The FT-IR spectra of AuNPs before (blue line) and after (red line) conjugation of MIZ$\mathrm{H}$ (gray line). The stretch vibration of $\mathrm{N}-\mathrm{H}\left(3122 \mathrm{~cm}^{-1}\right), \mathrm{C}=\mathrm{O}\left(1676 \mathrm{~cm}^{-1}\right)$ and $\mathrm{C}-\mathrm{N}\left(1202 \mathrm{~cm}^{-1}\right)$ in the FT-IR spectra of 02PMIZ-AuNPs after the coupling reaction indicated the successful conjugation of imidazole ring to AuNPs. 

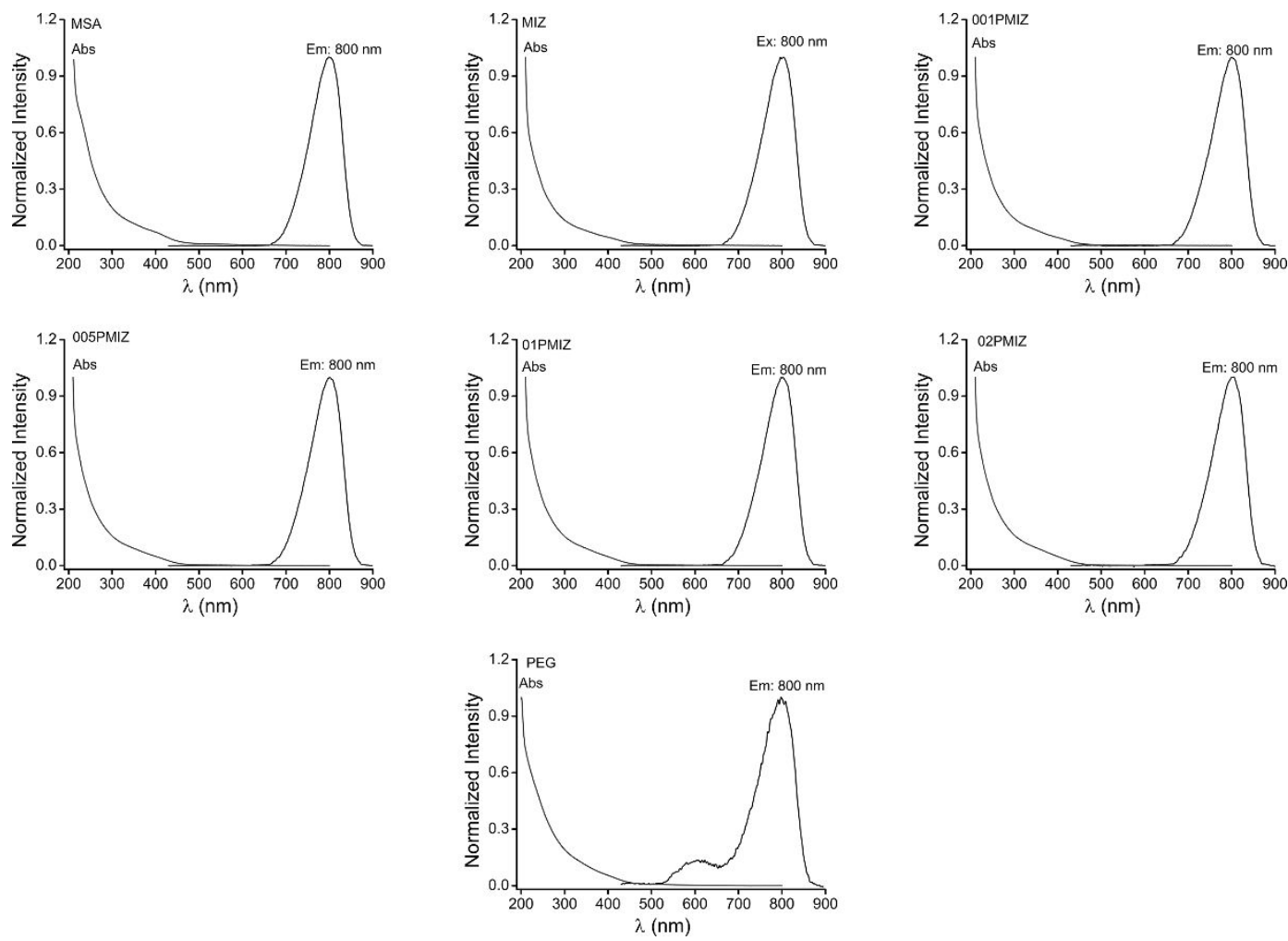

Figure S3. The absorption and emission spectra of the AuNPs. All the ultrasmall AuNPs (chargereversal AuNPs and non-charge-reversal AuNPs) exhibited strong near-infrared (NIR) emission with peaks at $\sim 800 \mathrm{~nm}$. The absorption spectra of these ultrasmall AuNPs were similar. All the AuNPs showed a similar broad strong UV absorption, and no surface plasmon absorption was observed at $\sim 520 \mathrm{~nm}$, which indicated the ultrasmall size of the AuNPs. 

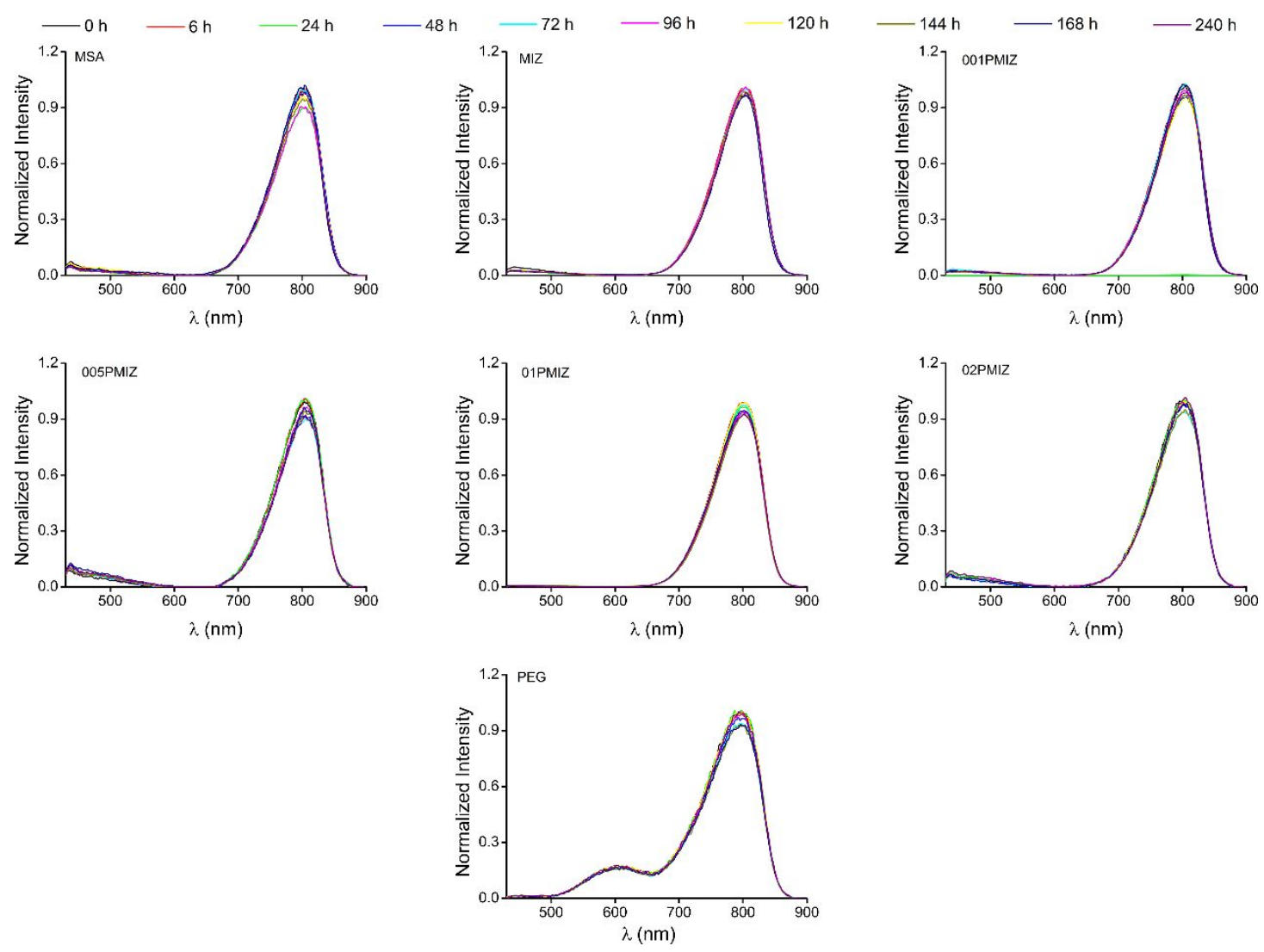

Figure S4. Fluorescence stabilities of AuNPs in DPBS at pH 7.4 after incubation for $240 \mathrm{~h}$. The luminescence intensities of all the AuNPs at $810 \mathrm{~nm}$ were almost unchanged over $240 \mathrm{~h}$ incubation, suggesting all AuNPs were highly stable under physiological environment.

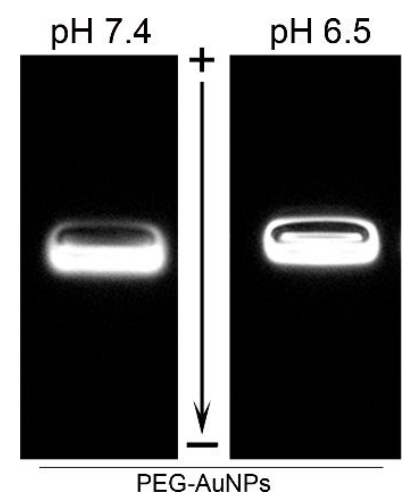

Figure S5. The agarose (1\%) gel electrophoresis of PEG-AuNPs. The PEG-AuNPs hardly migrate at pH 7.4 and 6.5, respectively, confirming the PEG-AuNP was neutral in the solution. 


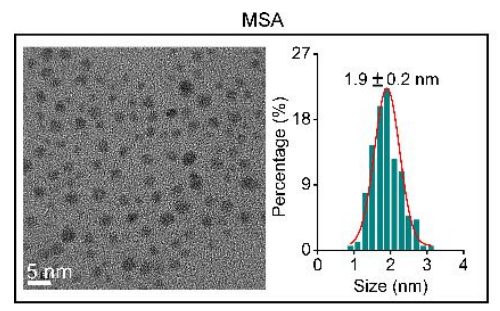

001PMIZ
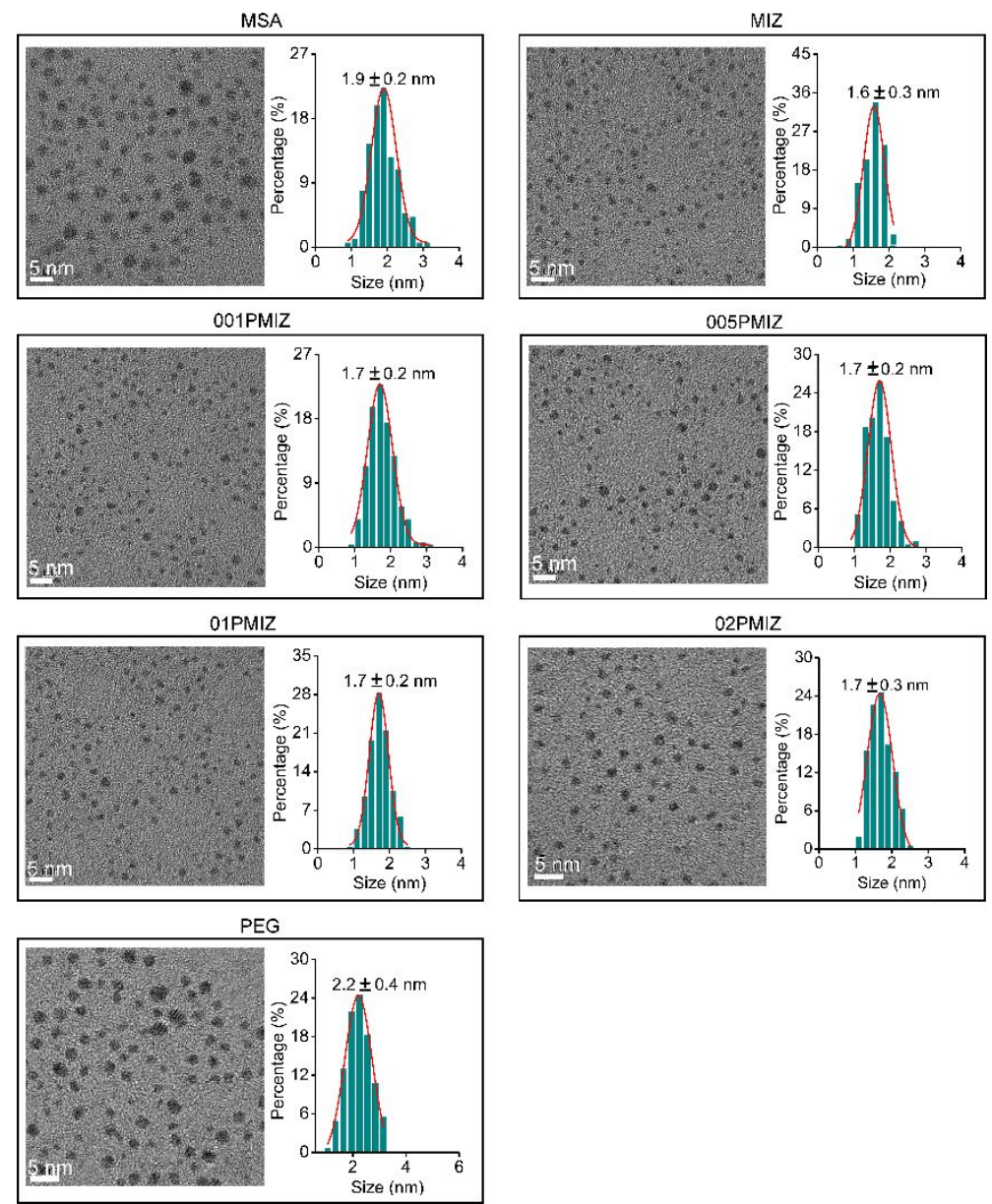

Figure S6. The typical TEM images and their corresponding core-size distributions of the AuNPs. The above results demonstrated that all the ultrasmall luminescent AuNPs showed very narrow size distributions with the identical core sizes of $1.7 \mathrm{~nm}$. 

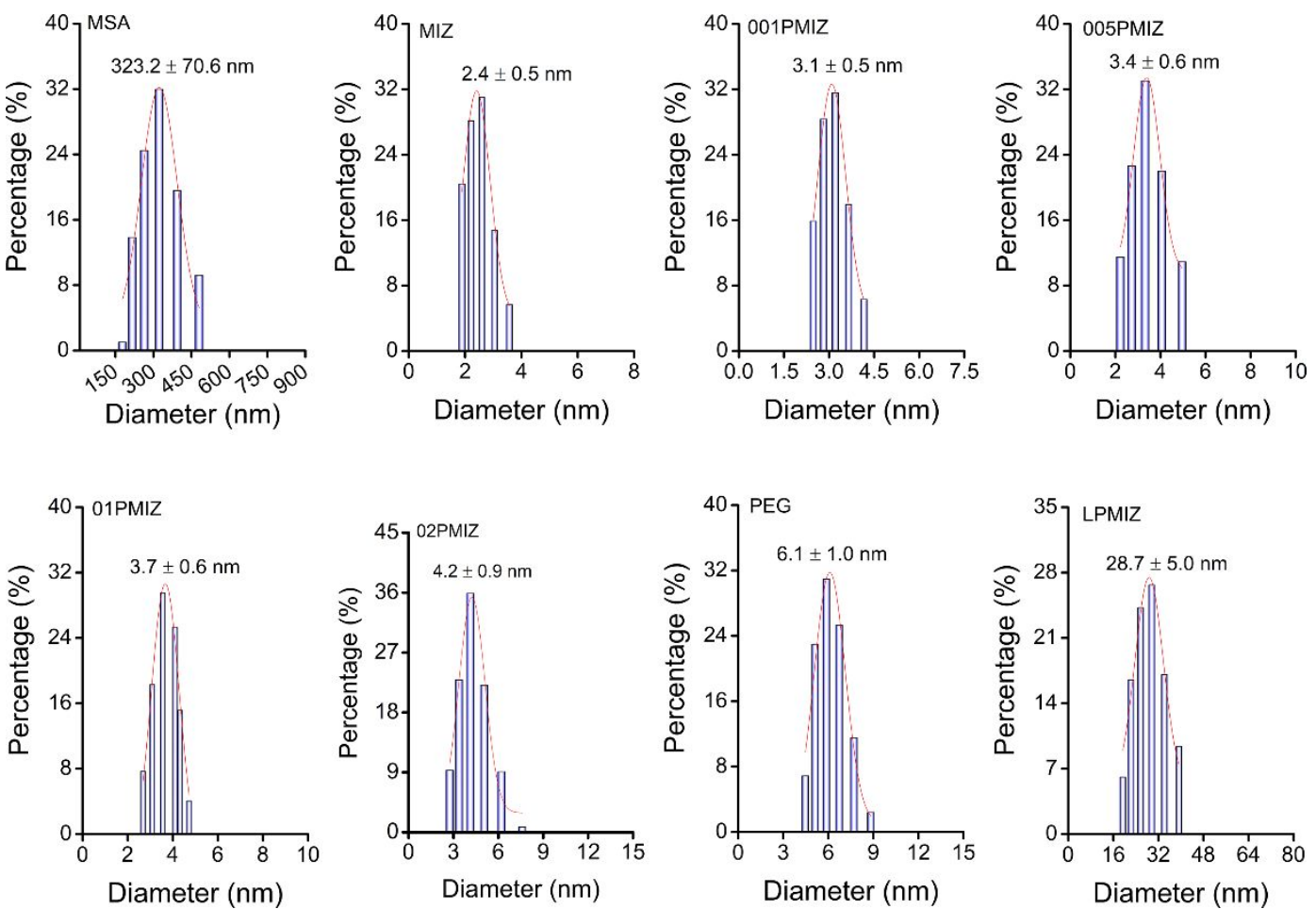

Figure S7. The HDs of AuNPs. The particle was dispersed in DPBS at pH 7.4 for the detection of diameter. The results showed the diameter of particles increased with the increasing density of PEG-SH, except MSA-AuNPs and LPMIZ-AuNPs. 

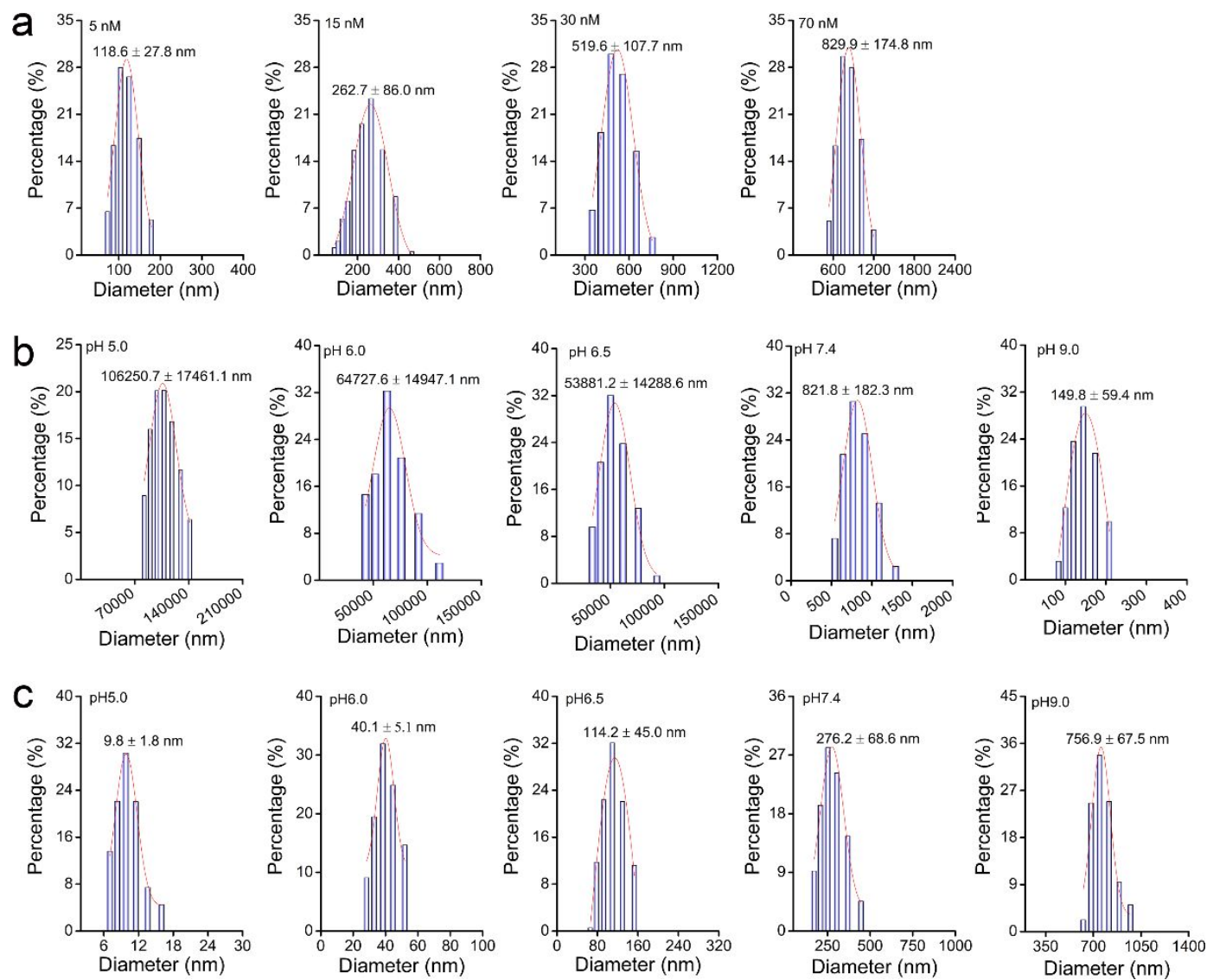

Figure S8. The HDs of MSA-AuNPs at different concentrations (a), $\mathrm{pH}$ values (b) and in the presence of $10 \% \mathrm{FBS}$ (c). The diameter was increased with the decreasing value of $\mathrm{pH}$ and the increasing concentration of particles, suggesting strong hydrogen bonding was formed between particles. After diluting into PB solution with 10\% FBS at different $\mathrm{pH}$, the diameter was decreased with the decreasing value of $\mathrm{pH}$, resulting from the broken hydrogen bonds among particles after binding FBS. The diameter of particles in PB solution with 10\% FBS at pH 7.4 was about $276 \mathrm{~nm}$, exceeding the cut-off limit of the organs interendothelial slits, such as liver, spleen and lung, thereby meaning high accumulation in liver, spleen and lung. ${ }^{2}$ 


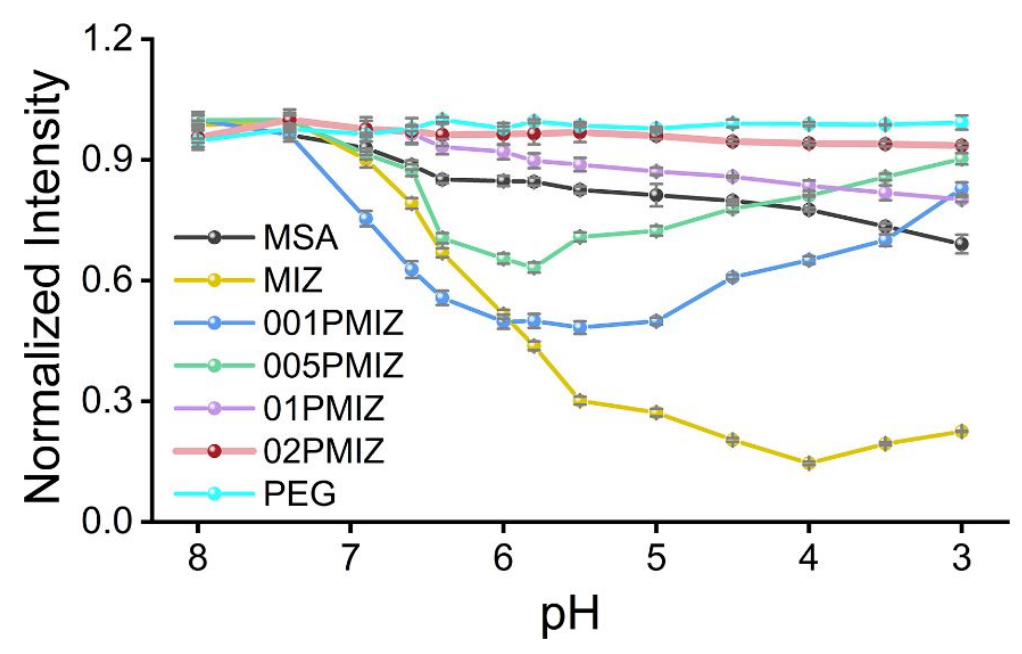

Figure S9. The luminescent intensity of the AuNPs at different pH values. The emissions at $800 \mathrm{~nm}$ of AuNPs with modification of low PEG-SH (MSA-AuNPs, MIZ-AuNPs, 001PMIZ-AuNPs and 005PMIZ-AuNPs) were more sensitive to $\mathrm{pH}$ changes, but those with modification high of PEG-SH (01PMIZ-AuNPs, 02PMIZ-AuNPs, and PEG-AuNPs) were unchanged at different $\mathrm{pH}$ values. The results indicated that the introduction of PEG-SH not only regulated the HDs and surface charge of the AuNPs, but also increased the optical stability of AuNPs at different $\mathrm{pH}$ values for feasible performance in the acidic environments. 

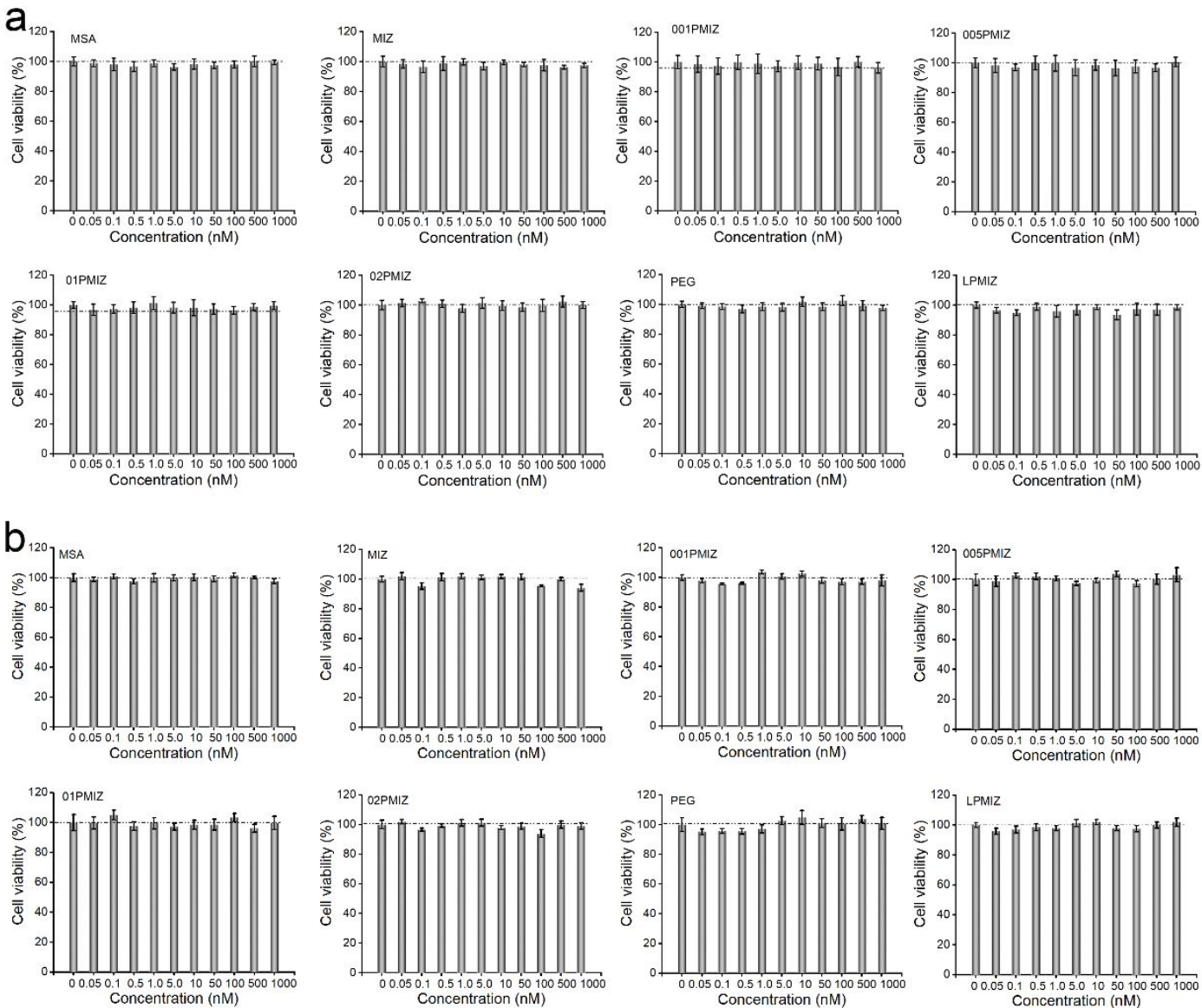

Figure S10. The cell viabilities of healthy human embryonic kidney 293T cells (a) and human breast cancer MDA-MB-231 cells (b) treated with AuNPs at different concentrations. The AuNPs incubated at different concentrations (from $0.05 \mathrm{nM}$ to $1000 \mathrm{nM}$ ) did not significantly influence the cell viabilities. Therefore, the investigated AuNPs showed negligible nanotoxicity to both normal and tumor cells. 


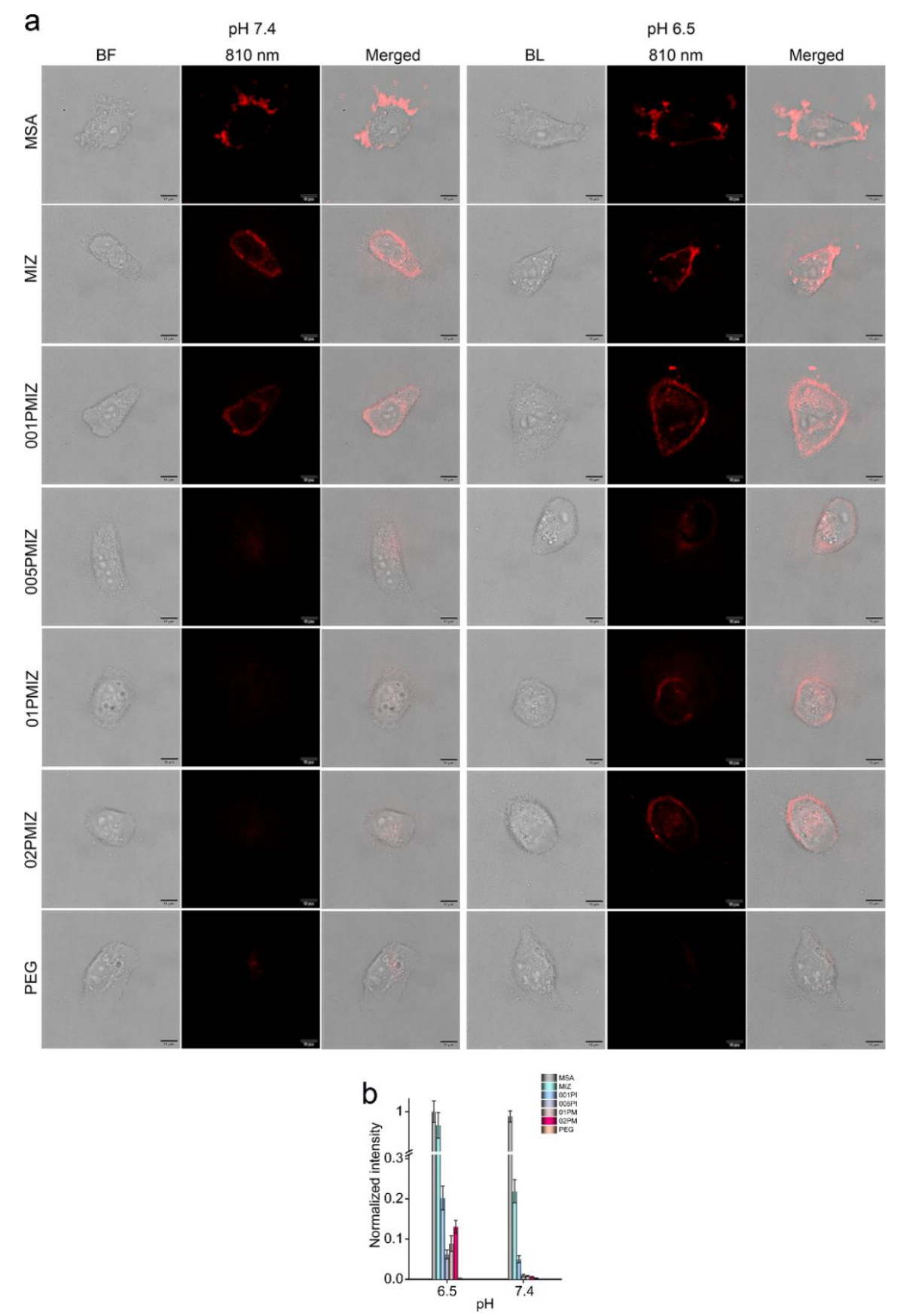

Figure S11. Membrane-binding imaging of the living cells treated with AuNPs. a, The fluorescent living cell images after incubated with AuNPs in DMEM supplemented with 10\% FBS for 10 min at pH 7.4 and 6.5, respectively, and their corresponding statistical luminescence intensities (b). Scale bar: 10 $\mu \mathrm{m}$. The intensities of cell membrane at $\mathrm{pH} 6.5$ were higher than those at $\mathrm{pH} 7.4$, resulting from the decreased electrostatic repulsion between particles and cell membrane. At $\mathrm{pH} 7.4$, the intensities of the charge-reversal AuNPs decreased with the increasing density of PEG-SH due to the enhanced resistance from the zwitterionic surface to the protein. At $\mathrm{pH}$ 6.5, the membrane intensity increased with the increasing $\zeta$-potential among 005PMIZ-AuNPs, 01PMIZ-AuNPs and 02PMIZ-AuNPs due to the increased electrostatic attraction between AuNPs and membrane. It should be noted that the intensities of MIZ-AuNPs and 001PMIZ-AuNPs at pH 6.5, increased significantly more than those at $\mathrm{pH} 7.4$, resulting from the $\mathrm{pH}$ induced aggregation or observable precipitation of the AuNPs at low $\mathrm{pH}$ values followed the charge-reversal behavior. Scale bar, $10 \mu \mathrm{m}$. 

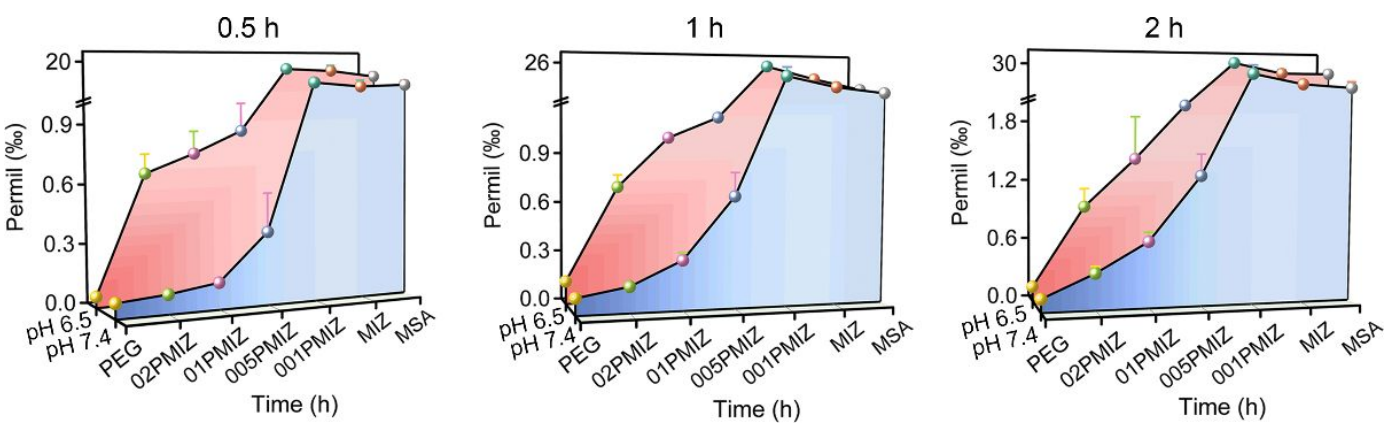

Figure S12. Cellular uptake of AuNPs at different pH values. The cell uptake ratios between $\mathrm{pH} 7.4$ and $\mathrm{pH} 6.5$ reached a high value (e.g., 6.7 times for 02PMIZ-AuNPs) after $0.5 \mathrm{~h}$ incubation, and decreased with extended incubation time, suggesting the charge-reversal AuNPs showed fast responses toward tumor acidic microenvironments. The gold concentrations were determined by ICP-MS. 
a
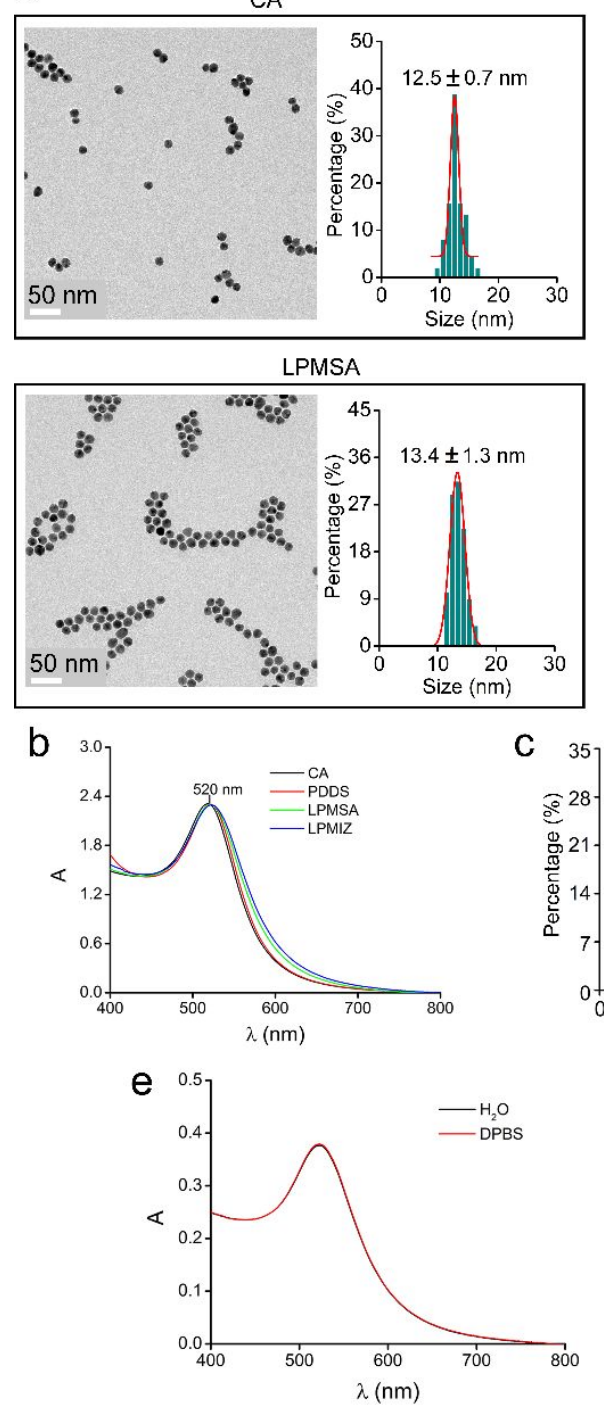

PDDS

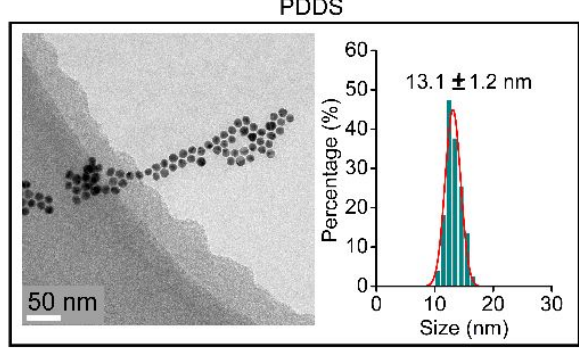

LPMIZ

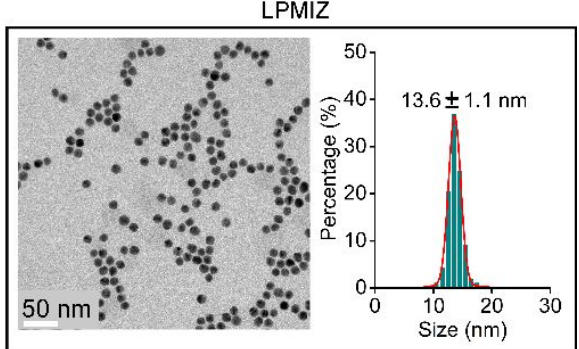

$\mathrm{d}$ pH $7.4+\mathrm{pH} 6.5$
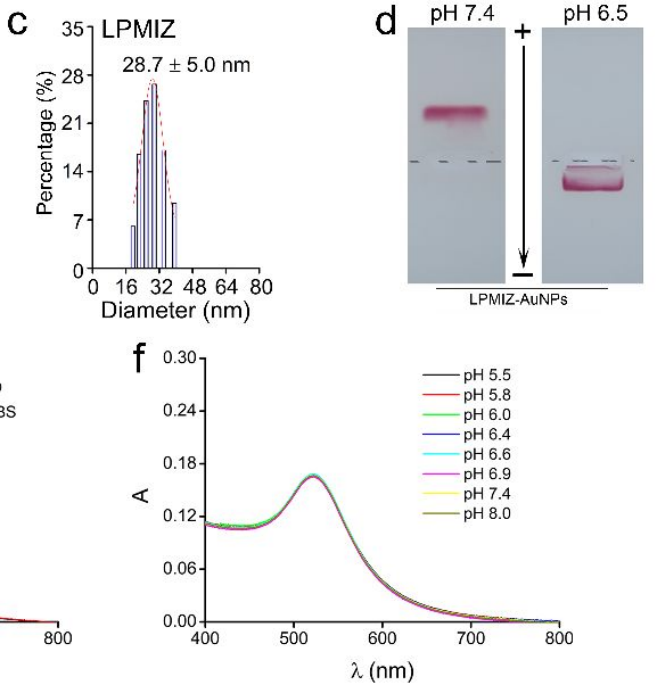

Figure S13. The characterization of the LPMIZ-AuNPs. The AuNPs with core size of $13 \mathrm{~nm}$ and similar surface chemistry (LPMIZ-AuNPs) was synthesized as the following method. Firstly, the citrate coated $13 \mathrm{~nm}$ AuNPs (CA-AuNPs) were prepared with a previously reported method. ${ }^{3}$ The CA-AuNPs solution was then mixed with bis(p-sulfonatophenyl) phenylphosphine dihydrate dipotassium salt (2 $\mathrm{mg} / 10 \mathrm{~mL}$ ) and stirred at room temperature overnight to develop the stability AuNPs (PDDS-AuNPs), followed by introducing PEG-SH and MSA-SH (the ratio of Au-to-PEG-SH-to-MSA-SH was 1:0.2:3) and stirring overnight to achieve the - $\mathrm{COOH}$ functionalized AuNPs (LPMSA-AuNPs). The mixture was centrifuged at $21000 \mathrm{~g}$ for further purification, followed by the functionalization with MIZ-H similar as the above procedure. The solution (LPMIZ-AuNPs) was finally purified by centrifuging at $21000 \mathrm{~g}$ to remove unreacted materials and stored at $4{ }^{\circ} \mathrm{C}$ for further use. a, The typical TEM image of AuNPs. The size of CA-AuNPs (CA), PDDS-AuNPs (PDDS), LPMSA-AuNPs (LPMSA) and LPMIZ-AuNPs (LPMIZ) were $\sim 13 \mathrm{~nm}$, suggesting the groups functionalization during the synthesis do not change the core size of AuNPs. b, The identical absorption spectra among CANP-AuNPs, PDDSNP-AuNPs, LPMSA-AuNPs and LPMIZ-AuNPs further confirmed that the size has no change during the functionalization. c, The HD of LPMIZ-AuNPs. d, The gel electrophoresis of LPMIZ-AuNPs at pH 7.4 and 6.5, respectively. e, The absorption spectra of LPMIZ-AuNPs maintained well both in DPBS and $\mathrm{H}_{2} \mathrm{O}$, and even at different $\mathrm{pH}$ values (f), indicating the high stability of LPMIZ-AuNPs. 

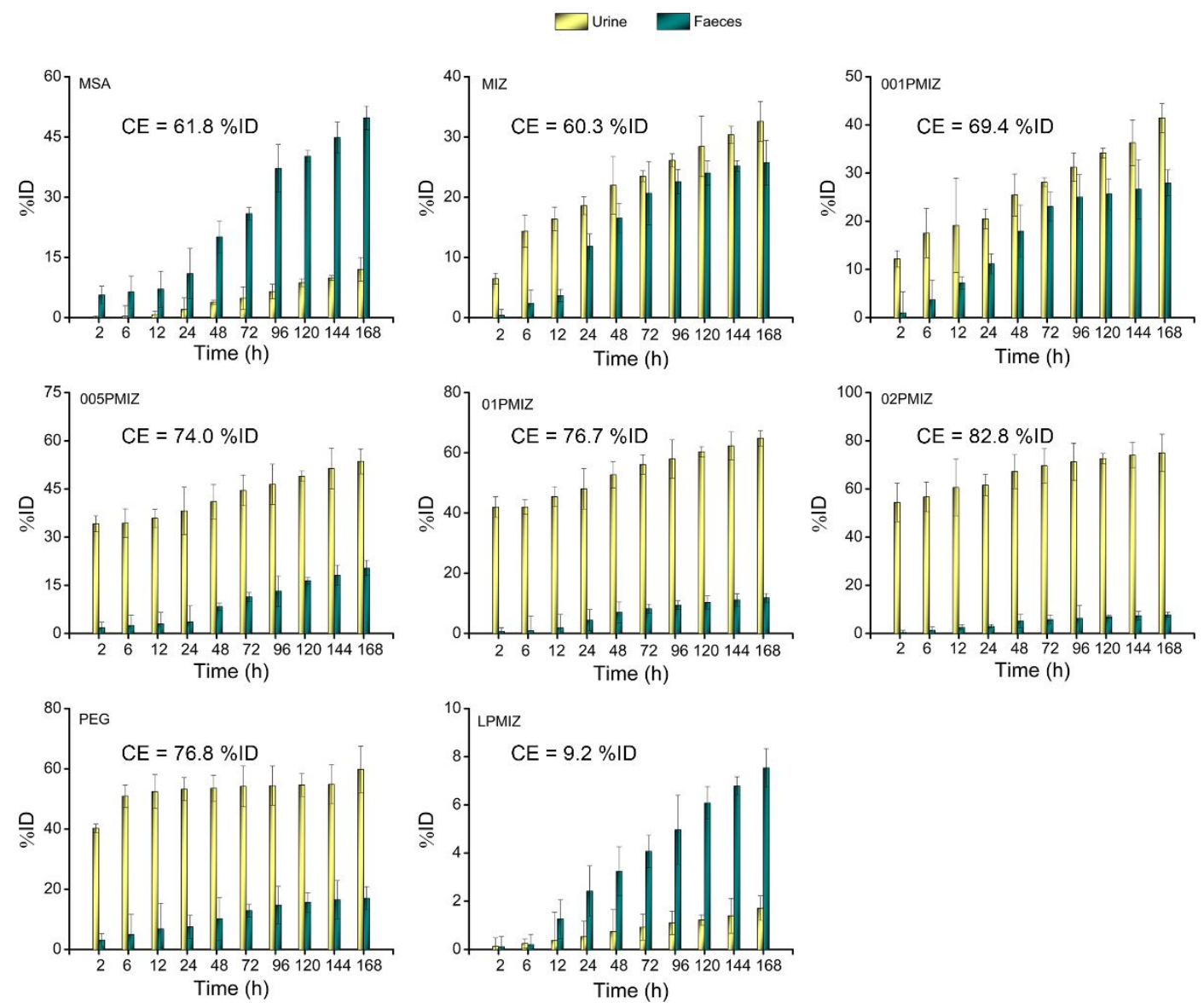

Figure S14. The renal and hepatobiliary clearance kinetics of the AuNPs. The total clearance efficiency (CE) was calculated from both urine and faeces after $168 \mathrm{~h}$ p.i.. The total CEs of all ultrasmall AuNPs reached 60.3-82.8 \% injection dose (ID), 6.7 times higher than those from the LPMIZ-AuNPs ( $9.2 \% \mathrm{ID})$, suggesting that elimination of the AuNPs depended highly on the core size rather than the HDs. According to a recent report that the biotransformation rate in liver decreased exponentially with the increase of the core size as observed from a series of PEGylated AuNPs with core sizes ranging from 5 to $100 \mathrm{~nm} .{ }^{4}$ The much higher rate of biotransformation from the AuNPs with small core sizes would cause higher total CEs. Therefore, the core size played vital role in governing their total CEs rather than HDs. 

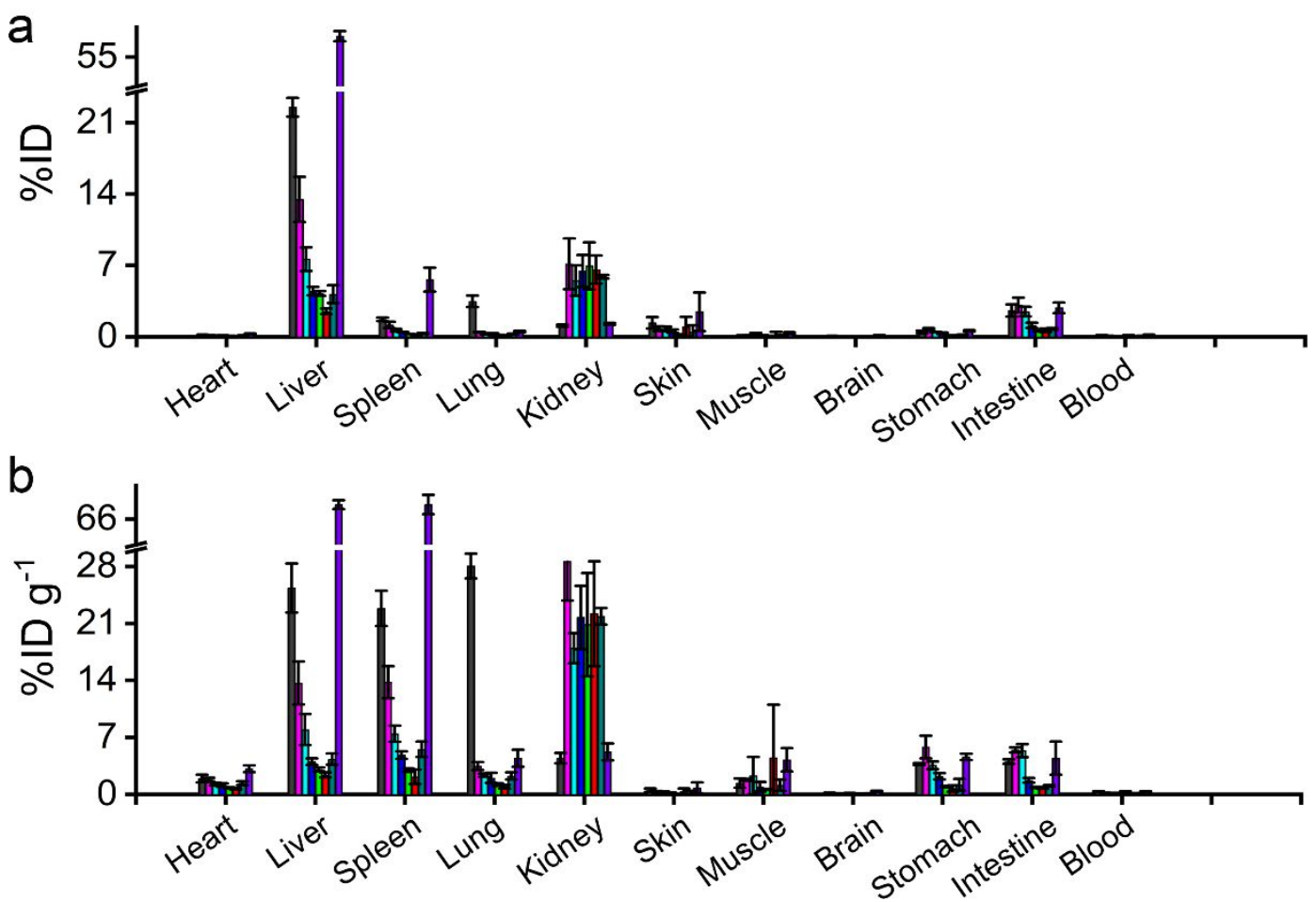

Figure S15. Biodistribution profiles of the AuNPs over 168 h p.i.. The ultrasmall AuNPs showed low accumulation in the main MPS organs such as liver ( 2.5 to $22.5 \%$ ID) and spleen $(\sim 0.2$ to $1.7 \%$ ID), in sharp contrast to those of the $13 \mathrm{~nm}$ LPMIZ-AuNPs with the high accumulation (72.4\%ID in liver and $5.6 \%$ ID in spleen). The ability of the ultrasmall charge-reversal AuNPs to escape from MPS organs increased with the increasing density of PEG-SH with a negligible 02PMIZ-AuNPs detected in liver (less than $2.5 \%$ ID) at $168 \mathrm{~h}$ p.i.. 


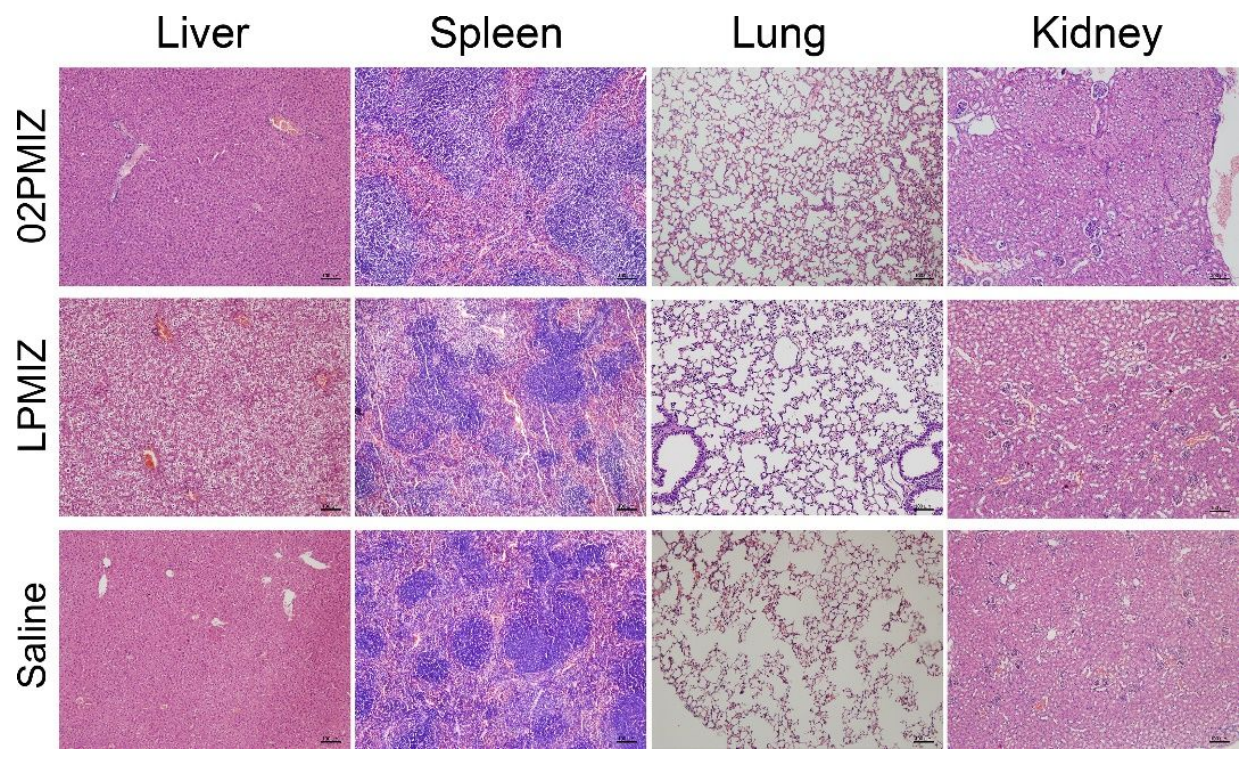

Figure S16. Histological images of the main organs after iv injection of AuNPs at 12 h p.i.. Compared with mouse iv injected with saline, the liver treated by LPMIZ-AuNPs showed significant lesions due to the high accumulation of the large AuNPs in the liver (72.4\%ID, at $168 \mathrm{~h}$ p.i.). However, the 02PMIZ-AuNPs had negligible toxicity with fast body clearance and extremely low accumulation in the MPS (2.5 \%ID in liver, at $168 \mathrm{~h}$ p.i.) organs. Scale bar, $100 \mu \mathrm{m}$.
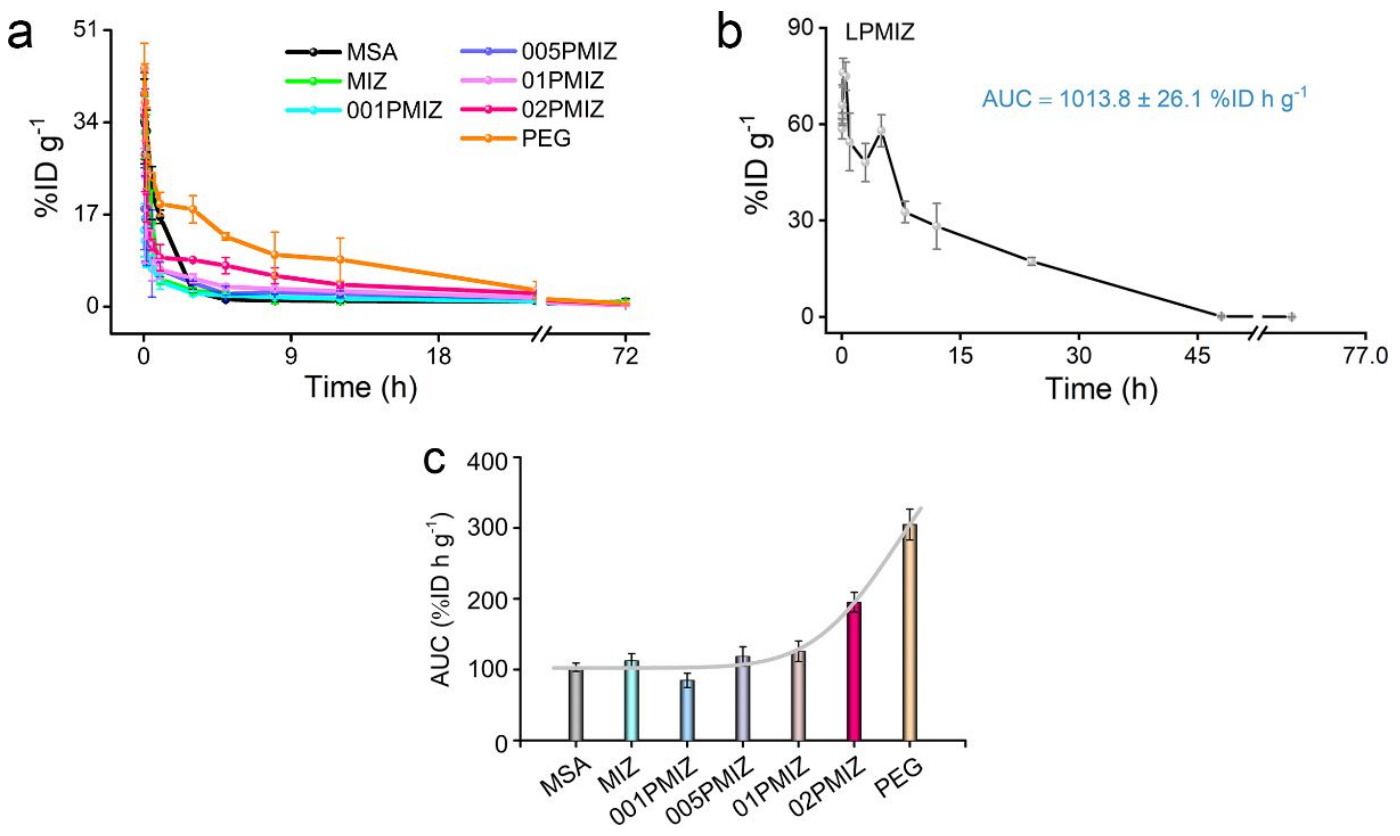

Figure S17. Pharmacokinetics of the AuNPs. a,b, Pharmacokinetics of the ultrasmall AuNPs (a) and LPMIZ-AuNPs (b). c, The area under the curve (AUC) for the ultrasmall AuNPs. With the increasing density of PEG-SH, the area under the curve (AUC) of the ultrasmall AuNPs increased, indicating an increased blood circulation. The AUC of LPMIZ-AuNPs was about $1013.8 \% \mathrm{ID} \mathrm{h} / \mathrm{g}$, much higher than those of the ultrasmall AuNPs. 

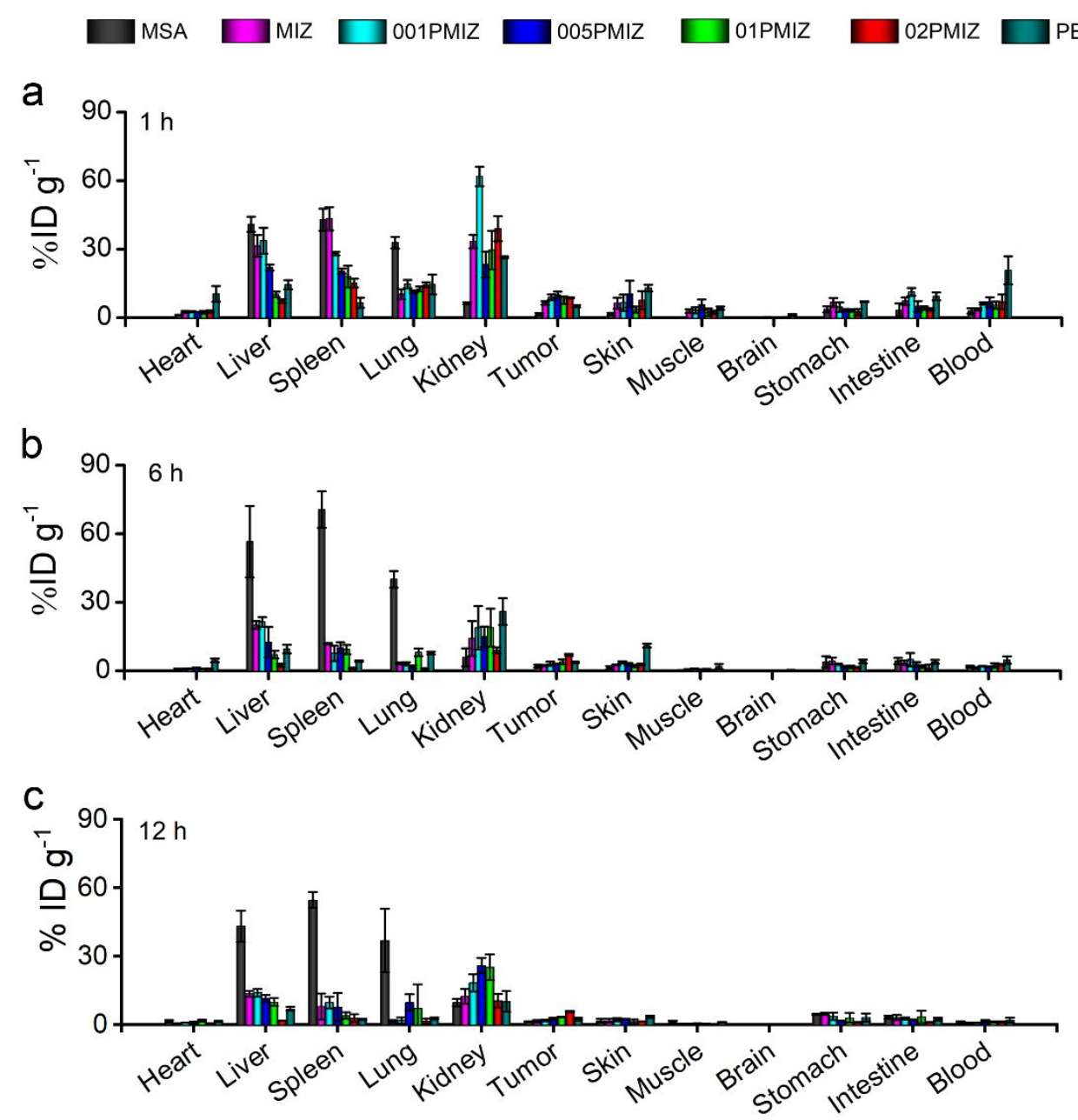

Figure S18. Biodistribution of AuNPs in mice with subcutaneous tumors at $1 \mathrm{~h}(\mathrm{a}), 6 \mathrm{~h}$ (b) and 12

h (c) p.i.. At $1 \mathrm{~h}$ p.i., the tumor-targeting efficiencies of the charge-reversal AuNPs were $\sim 9 \% \mathrm{ID} / \mathrm{g}$, which were higher than those of the non-charge-reversal AuNPs (MSA-AuNPs, $1.5 \%$ ID/g; PEG-AuNP, $5.3 \% \mathrm{ID} / \mathrm{g}$ ). With the increasing of PEG-SH, the accumulation in liver of charge-reversal AuNPs decreased to $2.5 \% \mathrm{ID} / \mathrm{g}$ (02PMIZ-AuNPs, at $6 \mathrm{~h}$ p.i.), but the tumor-targeting efficiency still maintained $6.9 \% \mathrm{ID} / \mathrm{g}$ (02PMIZ-AuNPs, at $6 \mathrm{~h} \mathrm{p.i),} \mathrm{significantly} \mathrm{higher} \mathrm{than} \mathrm{those} \mathrm{of} \mathrm{the} \mathrm{charge-reversal} \mathrm{AuNPs}$ with low amount of PEG-SH (MIZ-AuNPs, $2.3 \% \mathrm{ID} / \mathrm{g}$; 001PMIZ-AuNPs, $3.1 \% \mathrm{ID} / \mathrm{g}$ ) at $6 \mathrm{~h}$ p.i. Those results demonstrated that the regulation of the PEGylation of the charge-reversal AuNPs could not only enhance the tumor-targeting efficiency, but also decrease the liver nonspecific accumulation to achieve high tumor-targeting specificity. 

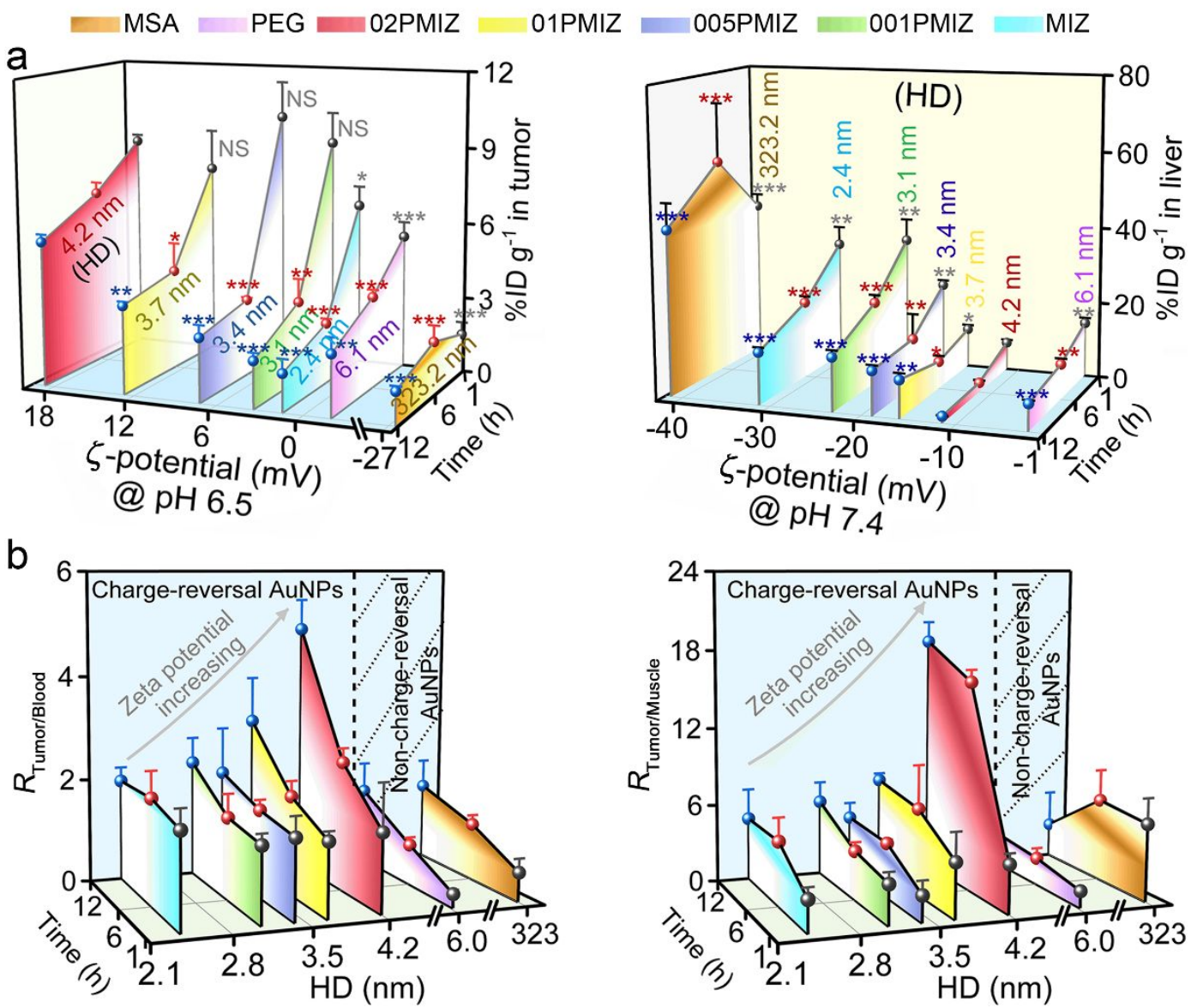

Figure S19. The relationship among biodistributions, $\zeta$-potential values and HDs of AuNPs. a, The time-dependent tumor-targeting efficiencies and liver accumulations over the $\zeta$-potential values of the AuNPs at 1, 6, $12 \mathrm{~h}$ p.i., respectively. Among the charge-reversal ultrasmall AuNPs, the tumor-targeting efficiency increased with the increase of the $\zeta$-potential values, while the accumulation in liver decreased. As control, the non-charge-reversal AuNPs showed lower tumor-targeting efficiency and higher liver accumulation. NS, no significance, ${ }^{*} P<0.05$; ${ }^{* *} P<0.01$; ${ }^{* * *} P<0.001$; Student's t-test. b, The ratio values of tumor-to-blood $\left(R_{\text {Tumor/Blood }}\right)$ and tumor-to-muscle $\left(R_{\text {Tumor/Muscle }}\right)$. Except the MSA-AuNPs, the ratio values of $R_{\text {Tumor/Blood }}$ and $R_{\text {Tumor/Muscle }}$ of the AuNPs increased over time. The ratio values of $R_{\text {Tumor/Blood }}$ and $R_{\text {Tumor/Muscle }}$ of charge-reversal particles increased with the increasing $\zeta$-potential values with 4.8 and 18.4 for 02PMIZ-AuNPs, at $12 \mathrm{~h}$ p.i, respectively, which were all higher than those of the corresponding non-charge-reversal AuNPs. 

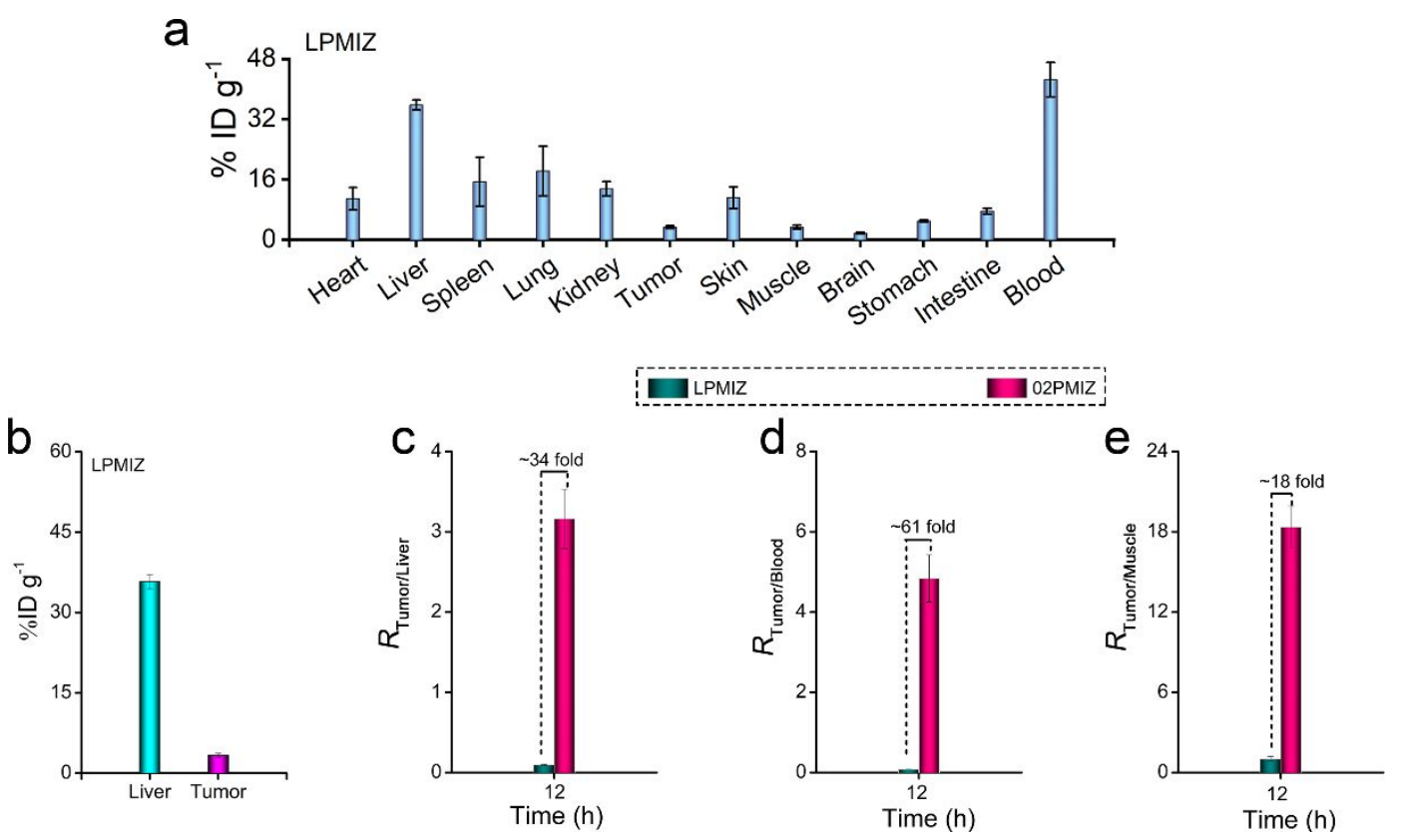

Figure S20. Biodistribution of the LPMIZ-AuNPs. a, The biodistributions in tumor and main organs of LPMIZ-AuNPs in mice with subcutaneous tumors at $12 \mathrm{~h} \mathrm{p.i..} \mathrm{b.} \mathrm{The} \mathrm{tumor-targeting} \mathrm{efficiency} \mathrm{and}$ liver accumulation of LPMIZ-AuNPs at $12 \mathrm{~h}$ p.i.. c-e, The ratios of tumor/liver (c), tumor/blood (d) and tumor/muscle (e) of the LPMIZ-AuNPs and 02PMIZ-AuNPs. These results indicated that the $13 \mathrm{~nm}$ LPMIZ-AuNPs showed low tumor-targeting efficiency $(3.4 \% \mathrm{ID} / \mathrm{g})$ and high liver accumulation (36.1\% $\% \mathrm{ID} / \mathrm{g})$, resulting in low tumor-targeting specificity $(\sim 0.1)$. 
a
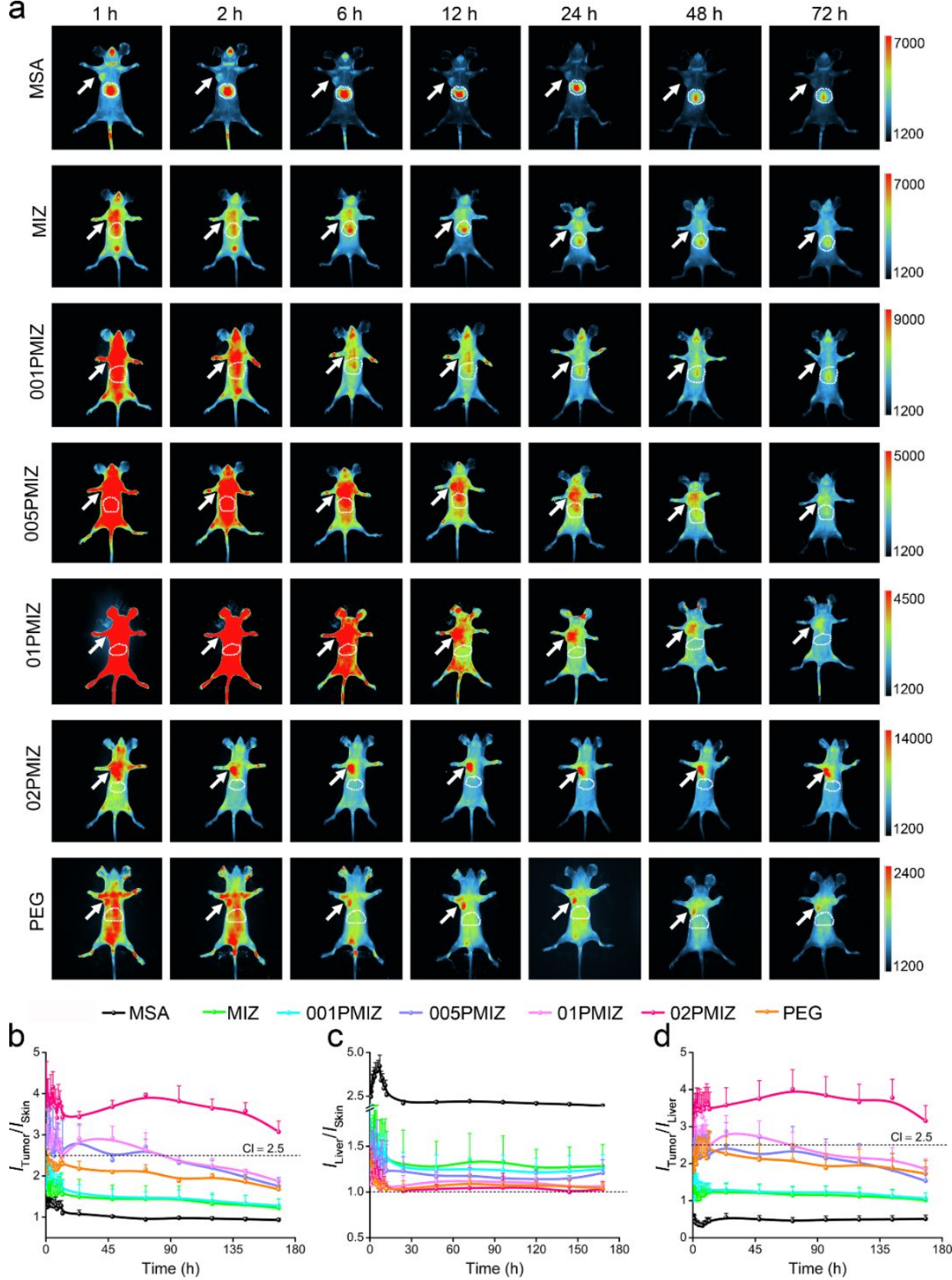

Figure S21. The in vivo imaging of the tumor targeting and liver accumulation of the AuNPs under short excitation wavelengths. a, In vivo imaging. The imaging parameters: Ex: 455-495 nm, Em: 800 $\mathrm{nm}$ longer, exposure time: $1 \mathrm{~min}$. The signal in normal skin was used as background intensity. b-d, The intensity ratio kinetics of tumor-to-skin $\left(I_{\text {Tumor }} / I_{\text {Skin }}\right)(\mathbf{b})$, liver-to-skin $\left(I_{\text {Liver }} / I_{\text {Skin }}\right)(\mathbf{c})$ and tumor-to-liver $\left(I_{\text {Tumor }} / I_{\text {Liver }}\right)(\mathbf{d})$. Under the excitation of short wavelengths, the tumor areas treated with 005PMIZAuNPs, 01PMIZ-AuNPs or 02PMIZ-AuNPs could reach a high contrast index (CI, S/N ratio) value (e.g., $\mathrm{CI}=2.5$ ), a general parameter for the evaluation of imaging quality. ${ }^{5}$ The targeting-specificity values $\left(I_{\text {Tumor }} / I_{\text {Liver }}\right)$ of 01PMIZ-AuNPs and 02PMIZ-AuNPs were also more than 2.5. The liver sites injected with 01PMIZ-AuNPs, 02PMIZ-AuNPs or PEG-AuNPs were showed much lower fluorescence signals as compare to those excited with longer wavelengths (600-645 nm, Figure 3a). The excitation with 455$495 \mathrm{~nm}$ wavelengths could be used to distinguish the superficial tumors rather than the tumors in the internal organs. Therefore, the longer wavelengths 600 to $645 \mathrm{~nm}$ were used as the excitation for obtaining the deep signals from the internal organs in the manuscript. 

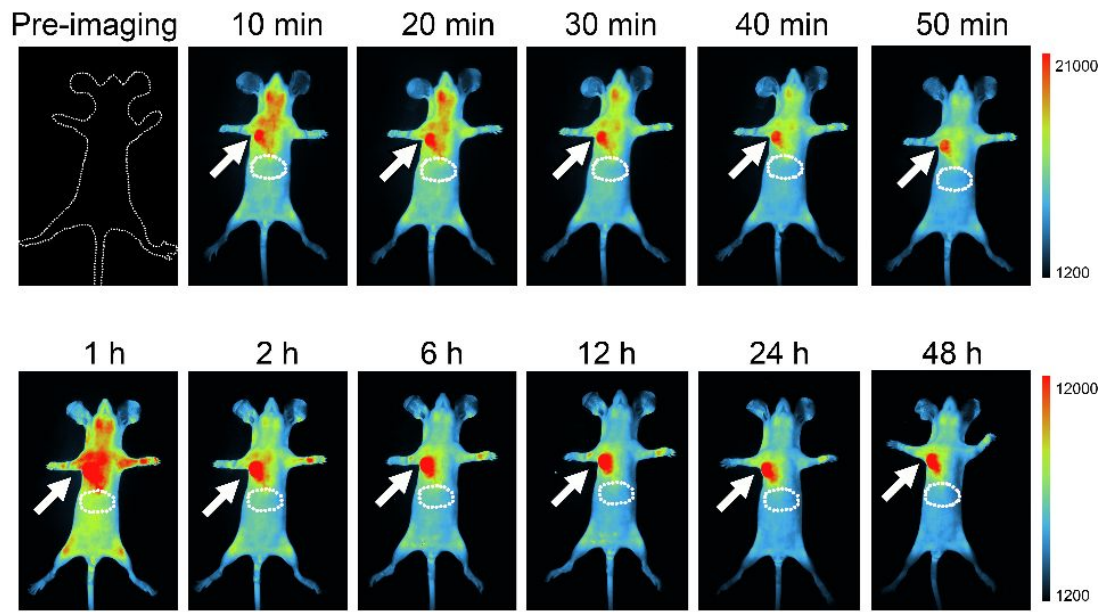

$48 \mathrm{~h}$
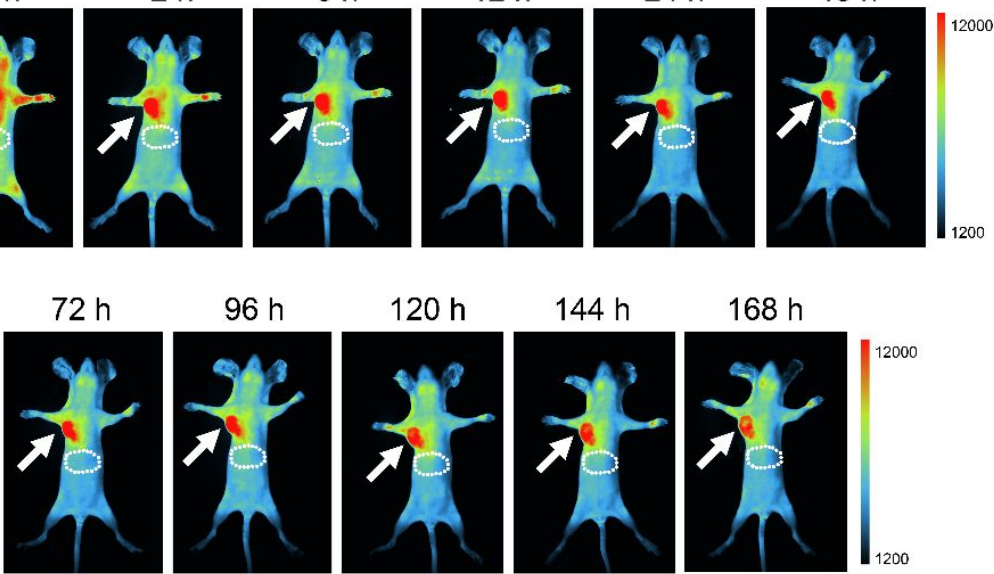

$168 \mathrm{~h}$

Figure S22. The in vivo tumor imaging of 02PMIZ-AuNPs. The imaging parameters: Ex: 455-495 nm, Em: $800 \mathrm{~nm}$ longer, exposure time: $1 \mathrm{~min}$. After iv injection of 02PMIZ-AuNPs, the tumor site was lighted rapidly and clearly at $10 \mathrm{~min}$ p.i., and maintained for $168 \mathrm{~h}$, suggesting that the charge-reversal AuNPs was extremely sensitive to tumor microenvironment with enhanced targeting efficiency and prolonged tumor retention time. 


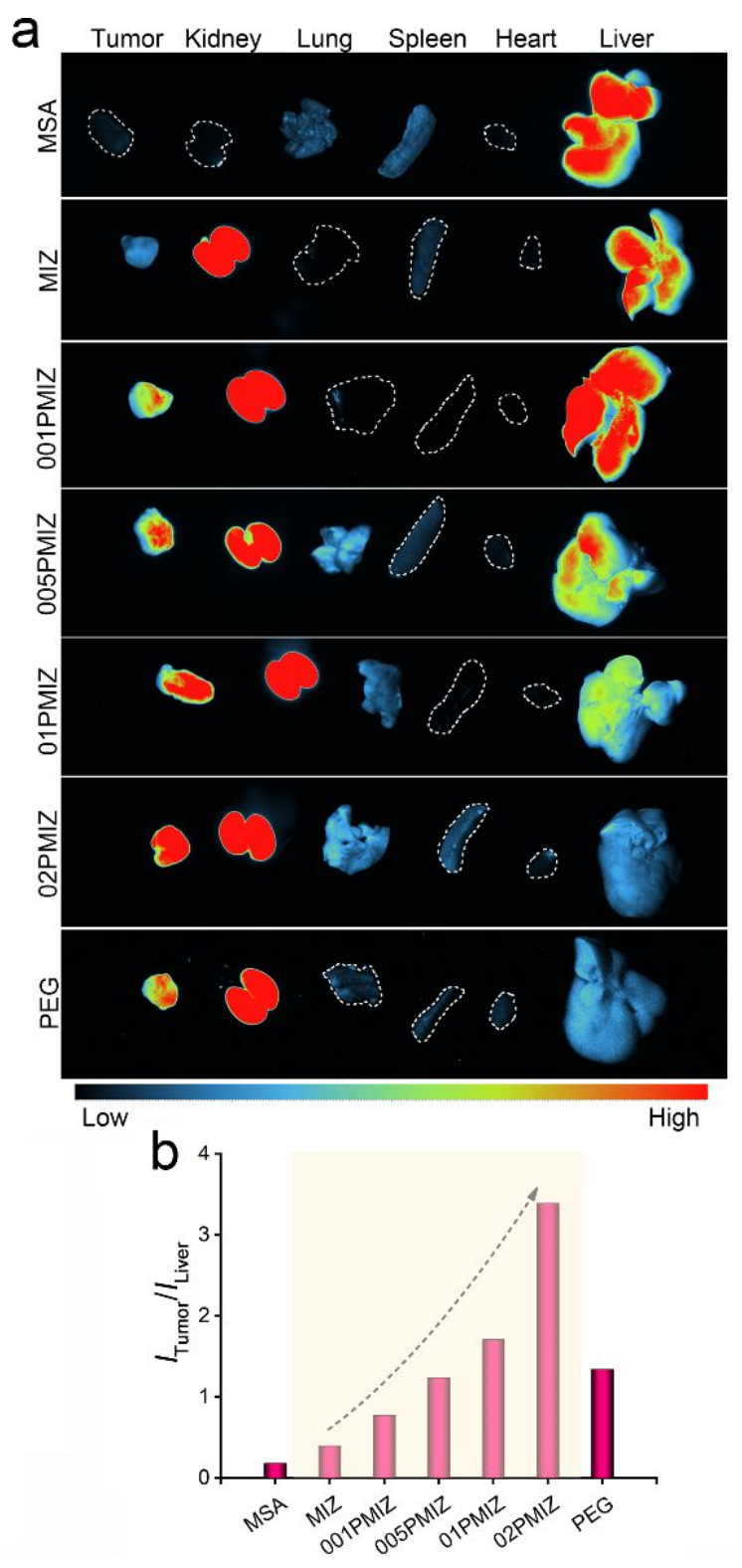

Figure S23. The ex vivo images of the tumor and main organs (a) and the corresponding statistical intensity ratio values of tumor-to-liver $\left(\boldsymbol{I}_{\text {Tumor }} / \boldsymbol{I}_{\text {Liver }}\right)(\mathbf{b})$. The imaging parameters: Ex: $455-495 \mathrm{~nm}$, Em: $800 \mathrm{~nm}$ longer, exposure time: $1 \mathrm{~min}$. Among the charge-reversal AuNPs, with increasing PEG-SH, the AuNPs showed enhanced ability to escape from MPS organs, and increased tumor targeting efficiency increased, resulting in increased ratio values of $I_{\text {Tumor }} / I_{\text {Liver. }}$. 

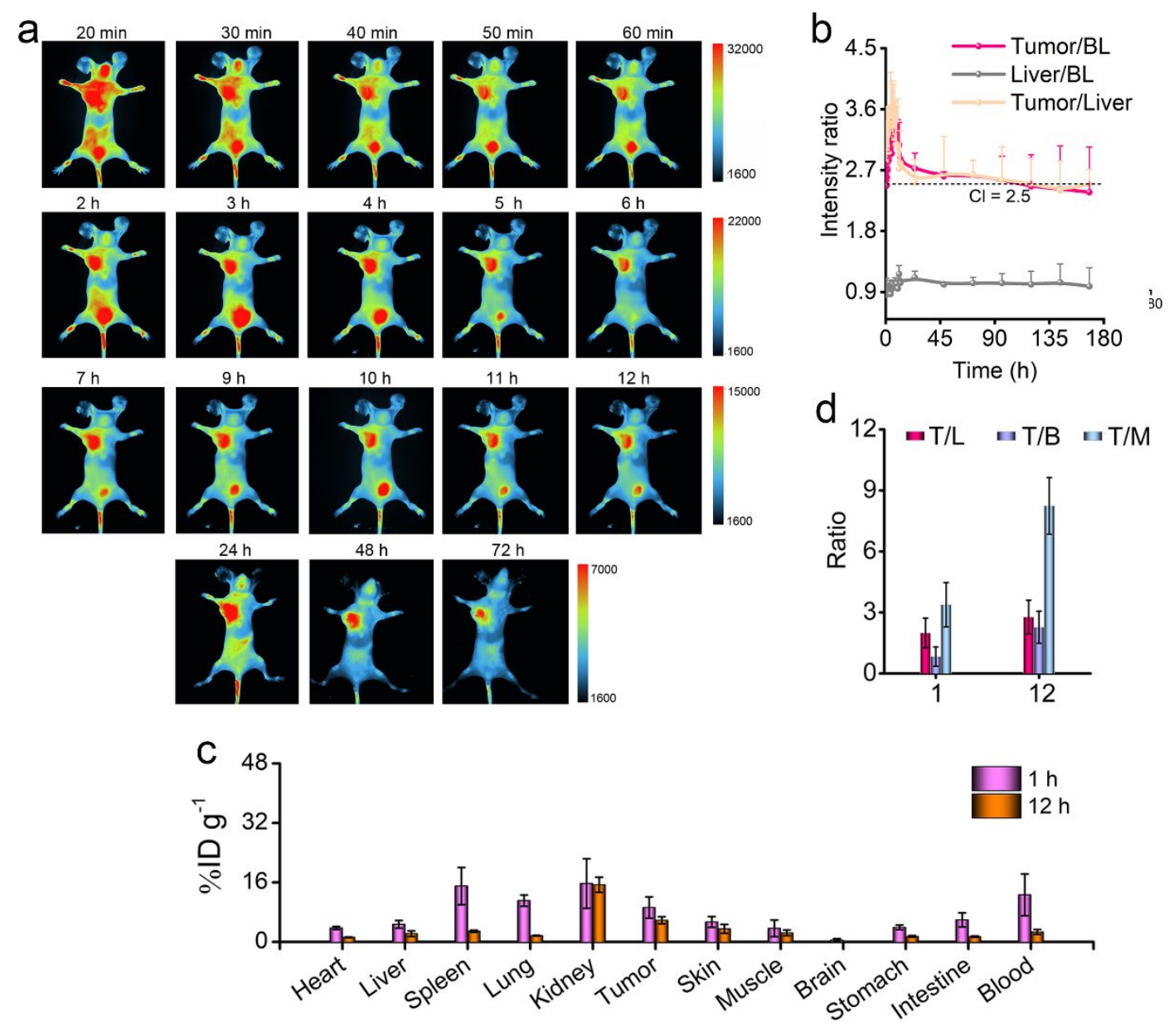

Figure S24. In vivo study of the orthotopic breast models. a, The in vivo images of orthotopic breast models after iv injection of 02PMIZ-AuNPs. The imaging parameters: Ex: 600-645 nm, Em: $800 \mathrm{~nm}$ longer, exposure time: $1 \mathrm{~min}$. b, The intensity ratio kinetics of tumor-to-skin, liver-to-skin and tumor-toliver. $\mathbf{c}$, The biodistributions in tumor and main organs at 1 and $12 \mathrm{~h} \mathrm{p.i.} \mathrm{d}$, The biodistribution ratios of tumor-to-liver (T/L), tumor-to-blood (T/B) and tumor-to-muscle (T/M). These results demonstrated that this optical nanoprobe could also be applied to the sensitive imaging of orthotopic breast tumors. 
a

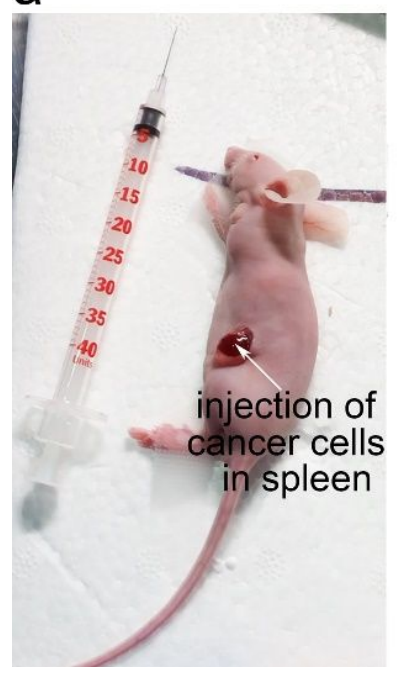

b

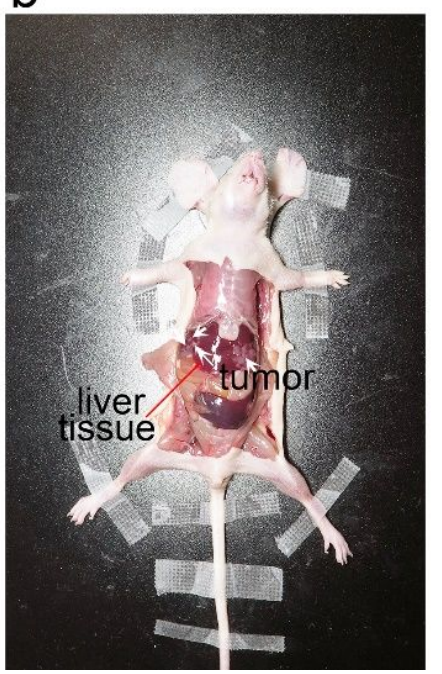

C

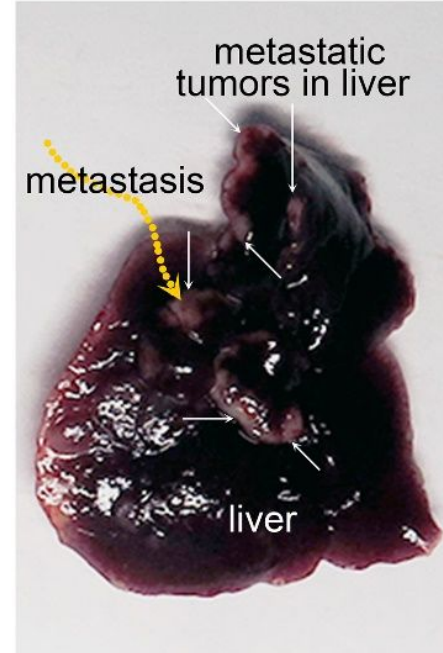

Figure S25. Liver metastasis model. a, The DMEM medium $(50 \mu \mathrm{L})$ containing $1 \times 10^{6} 4 \mathrm{~T} 1$ cancer cells was injected into the spleen. $\mathbf{b}$, The metastatic tumors were grown in the liver tissues in $\sim 10$ days. c, Harvested liver with metastatic tumors.
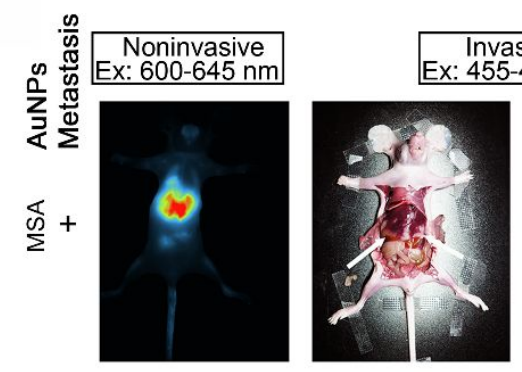

$5-495 \mathrm{~nm}$
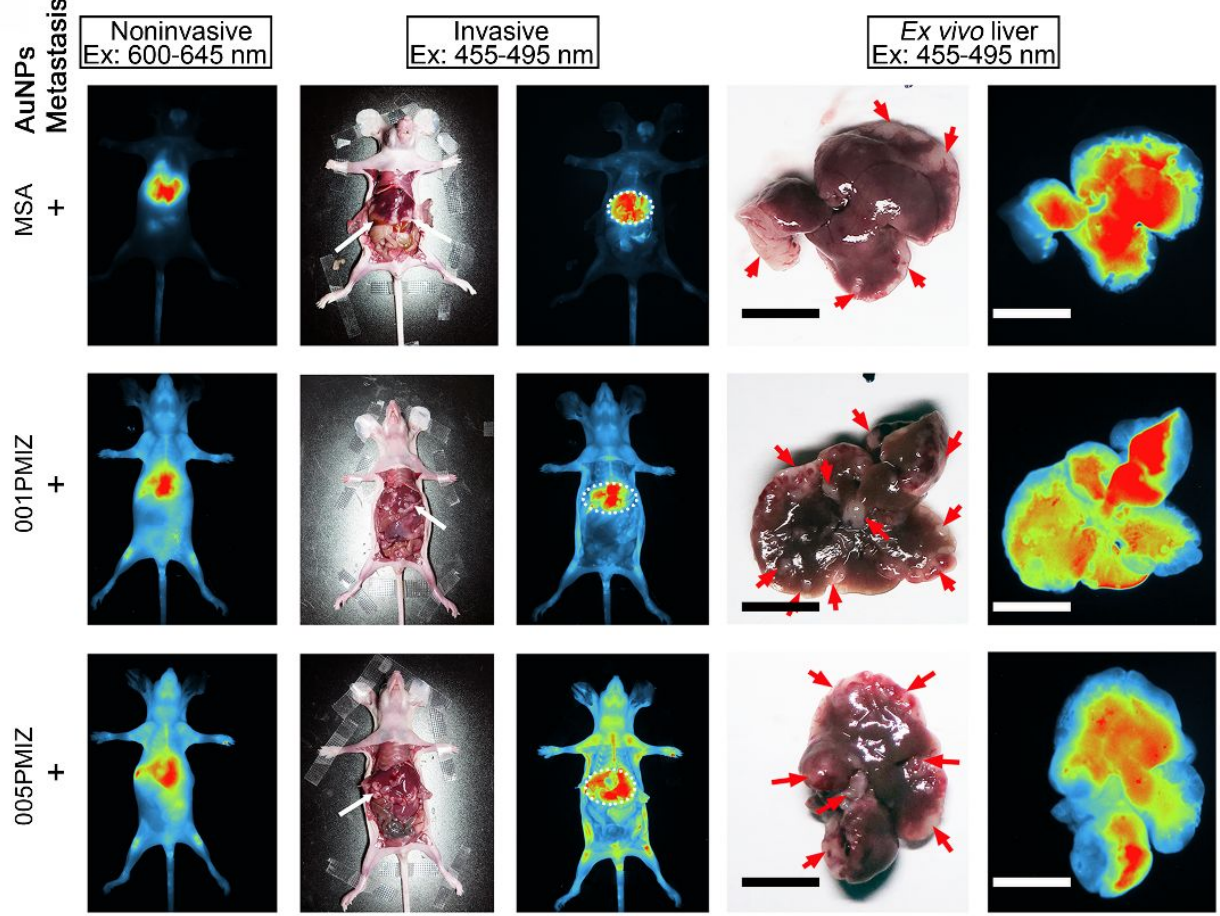

Low

High

Figure S26. Control groups for the identification of small metastatic tumors in liver. The noninvasive and invasive whole-body and liver tissue fluorescence images of liver-metastasis-bearing mice after iv injection of AuNPs. The arrow shows metastatic regions. The control groups including 001PMIZ-AuNPs, 005PMIZ-AuNPs, MSA-AuNPs, MIZ-AuNPs, 01PMIZ-AuNPs and PEG-AuNPs (Fig. 5a) with relative low tumor-targeting specificities were unable to distinguish between the liver tissue and liver metastasis. Scale bar, $10 \mathrm{~mm}$. 

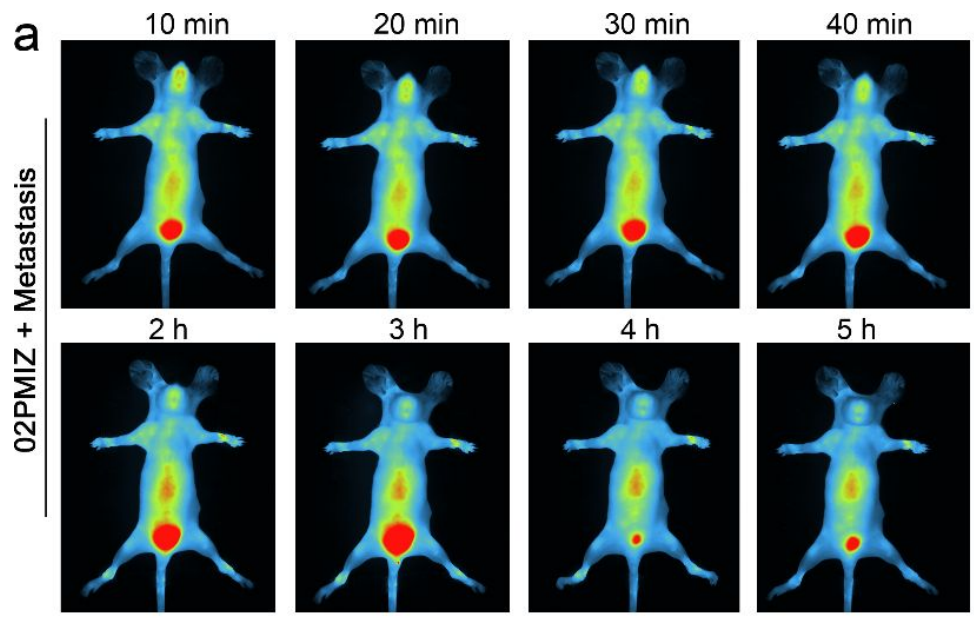

$3 \mathrm{~h}$

$4 \mathrm{~h}$

$5 \mathrm{~h}$
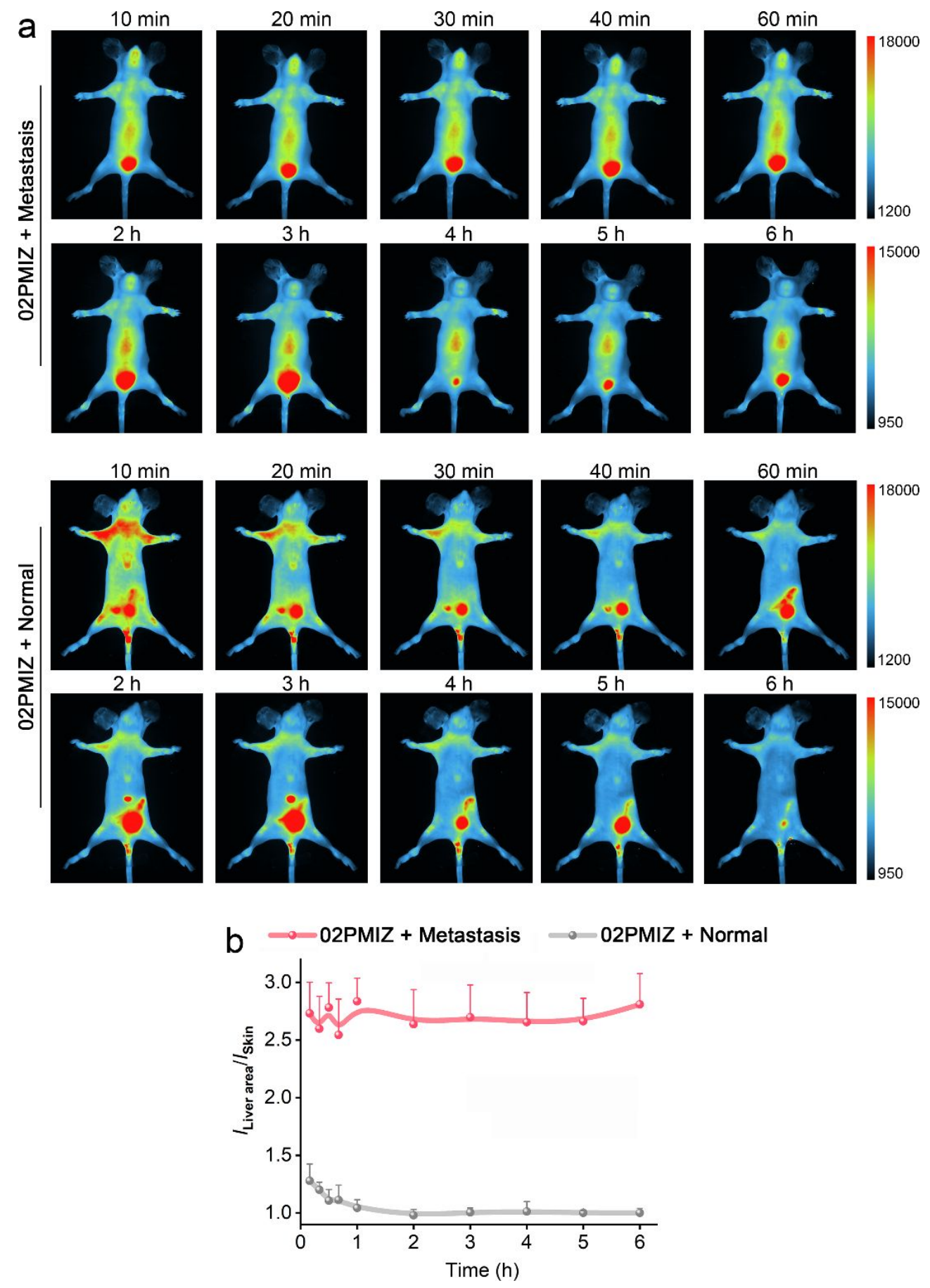

Figure S27. The imaging kinetics of the small cancer metastasis in liver. a, The time-dependent in vivo images of 02PMIZ-AuNPs in mice with liver metastasis and normal mice in $6 \mathrm{~h}$ p.i.. The imaging parameters: Ex: 600-645 nm, Em: $800 \mathrm{~nm}$ longer, exposure time: $1 \mathrm{~min}$. b, The intensity ratio of liver area-to-skin $\left(I_{\text {Liver area }} / I_{\text {Skin }}\right)$. In normal mice, the $I_{\text {Liver area }} / I_{\text {Skin }}$ was calculated to $\sim 1$, suggesting $02 \mathrm{PMIZ}$ AuNPs could escape from healthy liver tissue. Compared with the health mice group, the ratio values of $I_{\text {Liver area }} / I_{\text {Skin }}$ reached to $\sim 2.8$ for metastasis-bearing mice within $10 \mathrm{~min}$ p.i., indicating a fast and highquality imaging of the liver metastasis using 02PMIZ-AuNPs as nanoprobe. 

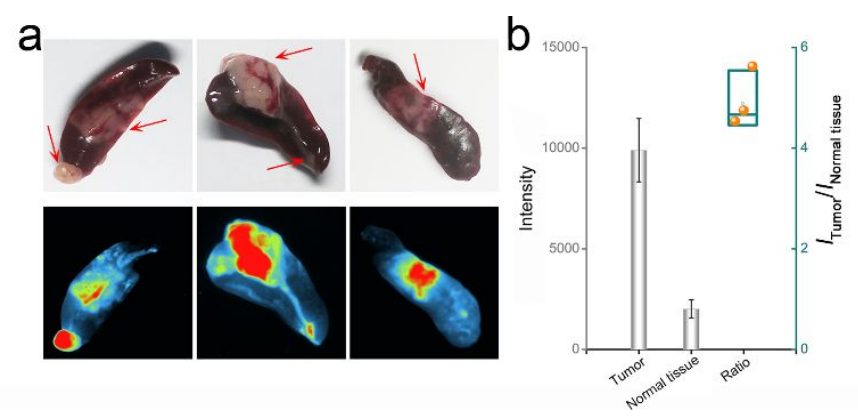

Figure S28. The imaging of primary tumor in spleen. a, The ex vivo image of the spleen with primary tumors $(N=3$, the arrow shows tumor region) at $6 \mathrm{~h}$ p.i.. The imaging parameters: Ex: $455-495 \mathrm{~nm}, \mathrm{Em}$ : $800 \mathrm{~nm}$ longer, exposure time: $1 \mathrm{~min}$. b, The fluorescence intensities of tumor region and normal spleen tissue, and the corresponding intensity ratio values $\left(I_{\text {Tumor }} / I_{\text {Normal tissue }}\right)$ as high as 5.2 . The results demonstrated that the primary tumors in the spleen were well identified due to the high tumor-targeting efficiency and extremely low accumulation of 02PMIZ-AuNPs in spleen. The primary tumor in spleen were obtained from the liver cancer metastasis model.
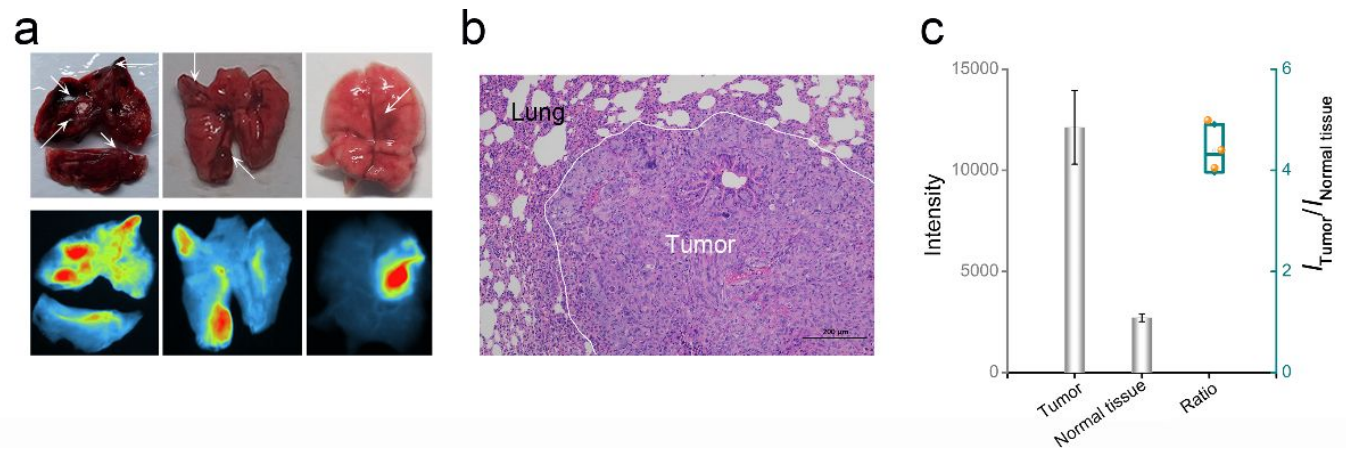

Figure S29. The imaging of cancer metastasis in lung. a, The ex vivo image of lung cancer metastasis $(N=3$, the arrow shows cancer metastatic region). The imaging parameters: Ex: 455-495 nm, Em: 800 $\mathrm{nm}$ longer, exposure time: $1 \mathrm{~min}$. b, Histological image of lung with metastatic tumor. c, The fluorescence intensities of tumor region and normal lung tissue, and the corresponding intensity ratio values ( $\left.I_{\text {Tumor }} / I_{\text {Normal tissue }}\right)$ as high as 4.5 . The results indicated that the developed optical nanoprobe could also be generalized to the sensitive imaging of lung cancer metastasis.

\section{Supporting Movie}

Movie S1. The 3D images of cell after stained 02PMIZ-AuNPs at pH 7.4 for 10 min.

Movie S2. The 3D images of cell after stained 02PMIZ-AuNPs at pH 6.5 for 10 min. 


\section{References}

(1) Zhou, C.; Hao, G.; Thomas, P.; Liu, J.; Yu, M.; Sun, S.; Öz, O. K.; Sun, X.; Zheng, J. J. A. C. I.

E. Near-Infrared Emitting Radioactive Gold Nanoparticles with Molecular Pharmacokinetics. Angew. Chem., Int. Ed. 2012, 51, 10118-10122.

(2) Blanco, E.; Shen, H.; Ferrari, M. Principles of Nanoparticle Design for Overcoming Biological Barriers to Drug Delivery. Nat. Biotechnol. 2015, 33, 941-951.

(3) Tan, Y.; Liu, L.; Wang, Y.; Liu, J. pH-Regulated Surface Plasmon Absorption from Ultrasmall Luminescent Gold Nanoparticles. Adv. Opt. Mater. 2018, 6, 1701324.

(4) Jiang, X.; Du, B.; Zheng, J. Glutathione-Mediated Biotransformation in the Liver Modulates Nanoparticle Transport. Nat. Nanotechnol. 2019, 14, 874-882.

(5) Liu, J.; Yu, M.; Ning, X.; Zhou, C.; Yang, S.; Zheng, J. PEGylation and Zwitterionization: Pros and Cons in the Renal Clearance and Tumor Targeting of Near-IR-Emitting Gold Nanoparticles. Angew. Chem., Int. Ed. 2013, 52, 12572-12576. 